\title{
GEOCHIMIE ET CONDITIONS DE CRISTALLISATION DES MINERAUX DU GROUPE DE L'HELVITE
}

\author{
Essaïd Bilal (*)
}

\begin{abstract}
Beryllium-bearing minerals (beryl, phenacite, helvite) are present in the quartz-muscovite and siderophyllite veins. In the albitites, only phenacite and helvite $(\mathrm{Fe}, \mathrm{Zn}, \mathrm{Mn})_{8} \mathrm{Be}_{6}\left(\mathrm{SiO}_{4}\right)_{6} \mathrm{~S}_{2}$ are found. Besides the Sucuri deposits, we studied also helvites from the northern skarn of Costabonne (France) and from quartz-wolframite veins of Dajishan (China). Paragenetic and geochemical data on helvites from these three occurences lead to propose a new structural formula:

$(\mathrm{Mn}, \mathrm{Zn}, \mathrm{Fe})_{8-x} \mathrm{Na}_{x} \mathrm{Be}_{6} \mathrm{Si}_{6} \mathrm{O}_{24} \mathrm{~S}_{2-\mathrm{x}} \mathrm{Cl}_{\mathrm{x}}$, analogous to that of haüyne.

To the standard substitutions $\mathrm{Zn}-\mathrm{Fe}-\mathrm{Mn}$ in helvite, we may add substitutions like Cl-S and Be-Li. Lithium probably replaces beryllium in the structure of helvite, the charge deficit being balanced by the entry of sodium. Crystal chemical constraints clearly play an important role for the incorporation of REE in helvite, but, in an alkaline context, helvite shows preference for heavy REE.

Danalite (the Fe end-member of helvite) is stable in a very limited domain of $\mathrm{fS}_{2}$ and $\mathrm{fO}_{2}$, which coincides with that of pyrrhotite. Genthelvite ( $\mathrm{Zn}$ end-member) is stable in a large domain of $\mathrm{fS}_{2}$ and $\mathrm{fO}_{2}$. On the other hand, helvite (Mn end-member) is stable in conditions of moderate $\mathrm{fO}_{2}$ and for a range of $\mathrm{fS}_{2}$ relatively large compared to that of genthelvite. In Sucuri,Goiás, Brazil, the ilmenites of the internal zone (where genthelvite is found) contain $16 \%$ of hematite, whereas those of the external zone contain no hematite. The zonations observed within the helvite crystals (genthelvite core and danalite border) reflect the variations of $\mathrm{fS}_{2}$ and $\mathrm{fO}_{2}$.

The compositional variations of the beryllium minerals observed in skarns may be explained by the following processes:

- either a late arrival of silica-rich solutions (high Si/Al ratio, presence of phenacite or helvite) within early formed aluminarich parageneses (musgravite, chrysoberyl),

- or a limited mobility of $\mathrm{Al}$ relatively to $\mathrm{Si}$, which would induce a limited extension of the aluminous parageneses (musgravite, chrysoberyl).

The activity of aluminium plays an essential role in the stability of the beryllium minerals: phenacite and/or helvite are stable when it is low, and beryl, chrysoberyl or musgravite when it is high. However, the increase of activity of other elements $(\mathrm{Ca}, \mathrm{Mn}$, $\mathrm{Fe}, \mathrm{Zn}$, alkalis) that would induce the formation of minerals incorporating alumina (garnet or felspars) would be unfavourable to the formation of beryl. Helvite is thus typically stable in calcic skarns, and it is associated with phenacite in the rocks submitted to alkaline metasomatism. Beryl, on the contrary, normally appears when acidic fluids separates from highly evolved granitic magmas (aplopegmatitic stage).

The zonation of helvite crystals in albitites (genthelvite core, danalite border) reflects the decrease of alkalinity of the fluids. This zonation on the crystal scale is also observed on the outcrop scale: helvite composition varies from $\mathrm{Ge}_{64} \mathrm{Da}_{10} \mathrm{He}_{26}$ in the internal zone to $\mathrm{Ge}_{23} \mathrm{Da}_{50} \mathrm{He}_{26}$ in the external zone. The same variation is observed in an albitized siderophyllite vein, from $\mathrm{Ge}_{25} \mathrm{Da}_{42} \mathrm{He}_{33}$ in a slightly albitized zone to $\mathrm{Ge}_{55} \mathrm{Da}_{39} \mathrm{He}_{22}$ in a highly albitized one. The formation of genthelvite is favoured, as compared with the other members of the helvite group, by alkaline conditions.

The compositional variations of helvite in the Sucuri massif would result from variations in $\mathrm{fS}_{2}$ and $\mathrm{fO}_{2}$ in the fluids. Genthelvite is stable under alkaline and oxydizing conditions.
\end{abstract}

\section{RESUMO}

Minerais de berílio (berilo, fenaquita e helvita) são constituintes de veios a quartzo-muscovita e veios de siderofilita. No albitito, somente a fenaquita e a helvita estão presentes. Este trabalho refere-se à ocorrência do maciço de Sucuri (Goiás, Brasil), às helvitas dos skarns de Costabonne, França e às helvitas de veios a quartzo-wolframita de Dajishan, China.

$\mathrm{O}$ estudo paragenético, geoquímico e experimental de helvitas $(\mathrm{Mn}, \mathrm{Zn}, \mathrm{Fe})_{8} \mathrm{Be}_{6} \mathrm{Si}_{6} \mathrm{O}_{24} \mathrm{~S}_{2}$ analisadas leva à uma nova definição da fórmula estrutural deste mineral que, por analogia àquela da hauyna é (Mn, $\mathrm{Zn}, \mathrm{Fe})_{8-\mathrm{x}} \mathrm{Na}_{\mathrm{x}} \mathrm{Be}_{6} \mathrm{Si}_{6} \mathrm{O}_{24} \mathrm{~S}_{2-\mathrm{x}} \mathrm{Cl}_{\mathrm{x}}$. Às substituições clássicas Zn-Fe-Mn, se ajuntam as substituições do tipo: Cl-S e Be-Li. O Li substitui o Be na estrutura da helvita, o déficit de carga elétrica resultante é compensado pela introdução de $\mathrm{Na}$ na estrutura. A substituição $\mathrm{Cl}-\mathrm{S}$ foi confirmada por estudo experimental. Restrições de ordem geoquímica desempenham um importante papel na introdução de terras raras na estrutura da helvita, de modo que num ambiente francamente alcalino, a helvita concentra mais facilmente as terras raras pesadas.

A danalita (pólo ferrífero da helvita) cristaliza-se em um domínio de fugacidade do enxofre e do oxigênio muito limitado e que corresponde ao domínio de estabilidade da pirrotita. A genthelvita (pólo zincífero da helvita) é estável em um extenso domínio de fugacidade do enxofre e oxigênio. Ao contrário, a helvita (pólo manganesífero da série) se desenvolve em um domínio de fugacidade de oxigênio moderado e de fugacidade do enxofre suficientemente extenso e comparável àquele da genthelvita.

A formação de mineralização de berílio de composição variável nos skarns resultaria, seja da sucessão de soluções tardias ricas em sílica (se a razão Si/Al é alta, a fenaquita ou a helvita se cristalizam) atuando sobre a paragênese aluminosa (musgravita, crisoberilo) formada precocemente, seja da fraca migração de Al em relação a Si. Neste último caso, a paragênese aluminosa (musgravita, crisoberilo) terá uma distribuição limitada. A atividade da alumina desempenha um papel essencial na variação da composição dos minerais de berílio. Se ela é fraca, a fenaquita e/ou helvita são estáveis e se ela é forte o berilo e o crisoberilo ou a musgravita são estáveis.

Entretanto, o aumento da atividade de alguns elementos ( $\mathrm{Ca}, \mathrm{Mn}, \mathrm{Fe}, \mathrm{Zn}$ ou alcalinos) tendendo a incorporarem alumina nas granadas ou nos feldspatos será um fator desfavorável para a formação do berilo. Assim, a helvita é um mineral tipicamente estável nos skarns cálcicos e se associa à fenaquita nas rochas submetidas ao metassomatismo alcalino. Por outro lado, o berilo aparece normalmente em condições onde um fluido ácido se separa de líquidos graníticos muito evoluidos (aplopegmatito).

A genthelvita é estável sob condições alcalinas e oxidantes.

(Traduzido pela redação) 


\section{INTRODUCTION}

L'helvite a pour formule générale $(\mathrm{Fe}, \mathrm{Zn}, \mathrm{Mn})_{8}$ $\mathrm{Be}_{6}\left(\mathrm{SiO}_{4}\right)_{6} \mathrm{~S}_{2}$ (Hassan \& Grundy 1985). Elle forme une solution solide entre trois pôles: helvite (Mn), danalite $(\mathrm{Fe})$ et genthelvite $(\mathrm{Zn})$. Ce groupe a une symétrie cubique et une structure de sodalite $\mathrm{Na}_{8} \mathrm{Al}_{6}\left(\mathrm{SiO}_{4}\right)_{6} \mathrm{Cl}_{2}$ (Hassan \& Grundy 1984, 1985). L'aluminium de la sodalite correspond au béryllium de l'helvite, $\mathrm{Na}$ au $\mathrm{Mn}, \mathrm{Fe}$ ou $\mathrm{Zn}$ et $\mathrm{Cl}$ à $\mathrm{S}$. La structure du groupe de l'helvite est caractérisée par six anneaux de quatre tétraèdres $\mathrm{BeO}_{4}$ et $\mathrm{SiO}_{4}$ correspondants aux plans (100) et (111). Ces anneaux sont enchaînés et forment un ensemble de canaux qui permettent l'entrée d'éléments non stoechiométriques.

Les données chimiques des minéraux du groupe de l'helvite dans la littérature (Glass et al. 1944; Beus 1962; Dunn 1976) suggèrent, d'une part qu'il y a miscibilité totale entre $\mathrm{Fe}$ et $\mathrm{Mn}$ et entre $\mathrm{Fe}$ et $\mathrm{Zn}$, et d'autre part qu'il n'y a pas de série complète entre $\mathrm{Mn}$ et $\mathrm{Zn}$. Dans la majorité des cas, les alcalins (Na et K) n'ont pas été reportés ou dosés, ce qui limite la discussion.

Les minéraux du groupe de l'helvite sont assez fréquents dans la nature. Deux facteurs essentiels déterminent leur apparition plutôt que celle d'autres silicates de béryllium tel que le béryl par exemple, il s'agit d'une faible activité de l'alumine et d'une forte activité de soufre accompagnée d'une forte activité de fer, du Mn ou du Zn dans la solution. L'acidité de la solution joue considérablement sur la fugacité du soufre (par l'intermédiaire des micas et des sulfures) donc sur la stabilité de l'helvite et du béryl.

Les différentes conditions de formation de ces minéraux expliquent la diversité de paragenèses dans lesquelles ils sont impliqués dans différents types de gisements (pegmatites, syénites, albitites, greisens et skarns). L'helvite et la danalite sont communes dans la nature alors que la genthelvite est relativement rare et que sa stabilité requiert des conditions particulièrement alcalines.

Une étude conjointe des minéraux du groupe de l'helvite et des minéraux associés devrait permettre d'interpréter en termes pétrogénétiques les différentes compositions de l'helvite. Pour cela, en plus du gisement de Sucuri, nous avons étudié les helvites des skarns Nord de Costabonne (France) et les helvites des veines à quartz et wolframite de Dajishan en Chine. Les moyens analytiques utilisés ont été: la microsonde, l'ICP et l'activation neutronique ainsi que l'étude des inclusions fluides sur les minéraux du groupe de l'helvite, le béryl et le quartz.

\section{ETUDE PARAGÉNÉTIQUE}

Du fait de la présence indispensable de $\mathrm{BeO}$, l'helvite apparaît généralement dans l'environnement granitique proche ou lointain. En fait, le minéral du bérylium courant dans les granites pegmatitiques eux mêmes est le béryl. L'helvite n'apparaît que dans les zones affectées par la circulation des fluides hydrothermaux qui produisent feldspathisation, greisen, veines et skarns.

\section{Gisement de Sucuri}

\section{Zones de feldspathisation}

Le gisement principal d'albitites est situé au NordEst du massif dans la carrière Tratex (Bilal 1991). Les filons d'albitites sont toujours localisés dans le granite à biotite à grain moyen (Fig. 1). L'albitisation efface la foliation du granite, elle est donc postérieure à la dernière phase tectonique (l'orogenèse Uruaçuano 1.3 - $1.1 \mathrm{Ga}$ ) qui affecte le massif de Sucuri d'âge 1.77 Ga. (Pimentel et al. 1990). Les zones albitisées se présentent soit en bandes métriques d'allure filonienne, soit en tâches irrégulières centimétrique dans le granite à biotite (Fig. 1). L'albitisation est progressive et détermine une zonation minéralogique de la périphérie au coeur du filon d'albitite à biotite (tableau 1) avec un changement de la composition de l'helvite.

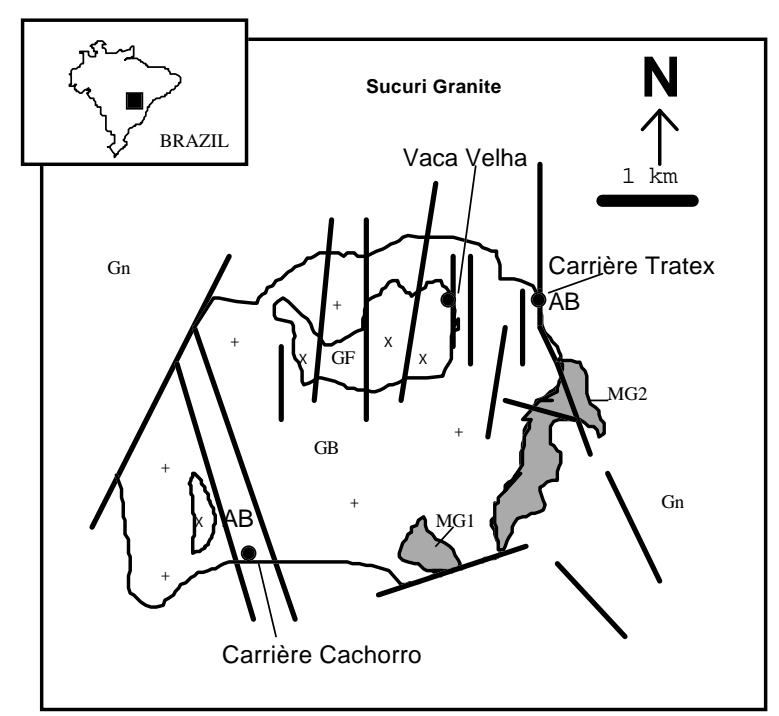

Figure 1 : Carte géologique du massif granitique de Sucuri (Goias, Brésil) Bilal (1991). Gn: gneiss;

$G B$ : granite à biotite; GF: granite fin;

$M G$ :microgranite; $A B$ : zones albitisées.

Figure 1: Geologic map of the Sucuri granitic massive (Goiás, Brazil).

La danalite (pôle ferrifère de l'helvite) apparaît dès la première zone caractérisée par la transformation partielle de la muscovite en albite. Elle est associée à la biotite, aux sulfures et parfois à la fluorine. La composition de la danalite varie entre $\mathrm{Ge}_{24} \mathrm{Da}_{51} \mathrm{He}_{25}$ et $\mathrm{Ge}_{23} \mathrm{Da}_{50} \mathrm{He}_{26}$.

La deuxième zone est caractérisée par la disparition complète de la muscovite. La danalite $\mathrm{Ge}_{30} \mathrm{Da}_{49} \mathrm{He}_{21}$ s'appauvrit légèrement en composant helvite $(\mathrm{Mn})$ par rapport à celle de la première zone.

La troisième zone et la quatrième zone sont marquées par le remplacement total du feldspath potassique par de l'albite. La danalite est zonée, elle 


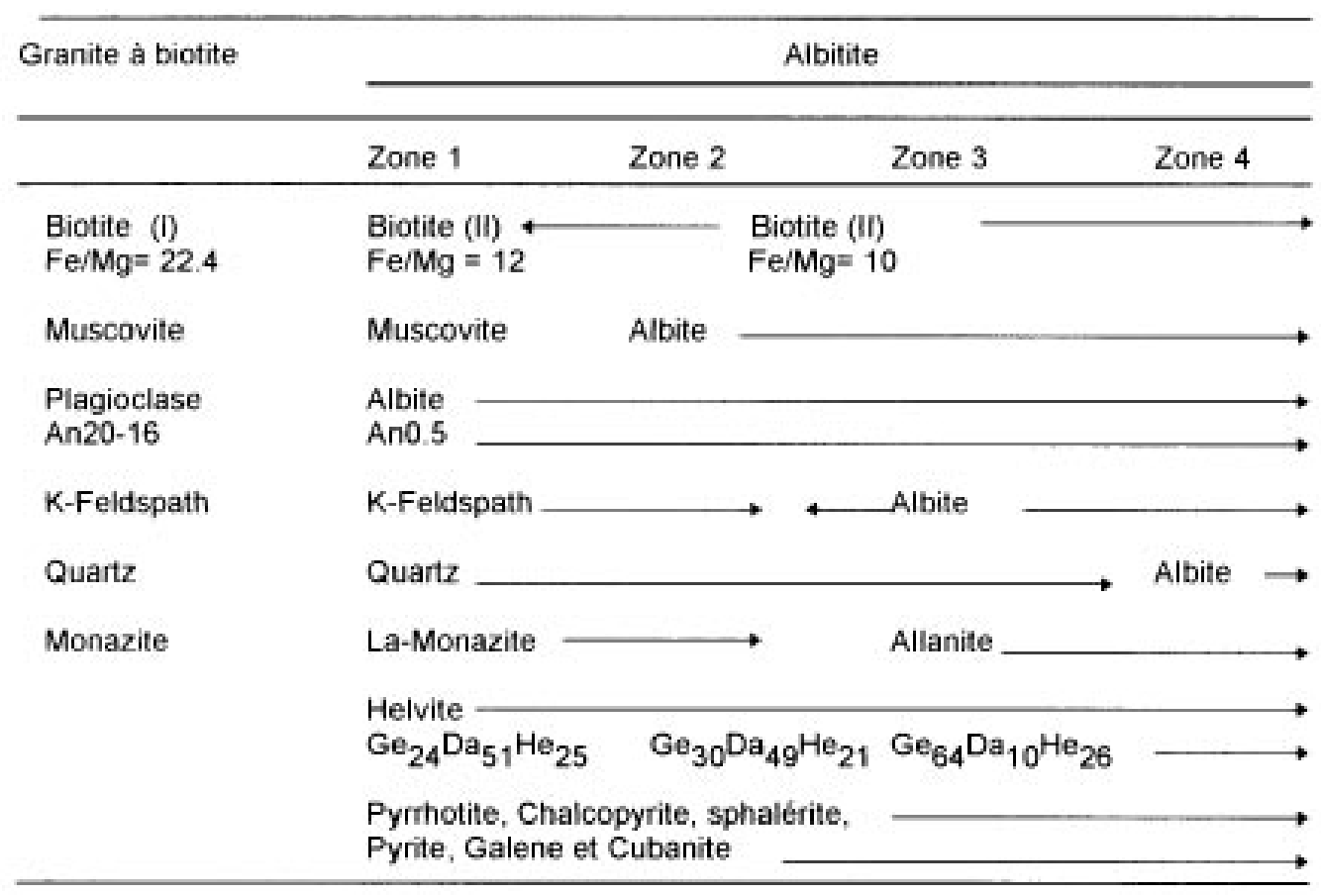

Tableau 1: Séquence d'apparaition des minéraux de différentes zones de l'albitite du massif de Sucuri. Table 1: Cristallization sequence of mineral from different zones of the Sucuri massive.

présente un coeur constitué de genthelvite $\mathrm{Ge}_{64} \mathrm{Da}_{10} \mathrm{He}_{26}$ et une bordure de composition de danalite $\mathrm{Ge}_{25} \mathrm{Da}_{52} \mathrm{He}_{22}$.

Des sulfures (pyrrhotite, chalcopyrite, sphalérite et galène) sont associés à l'helvite.

Les teneurs en $\mathrm{Na}_{2} \mathrm{O}$ sont assez élevées: $0.12 \%$ à $0.31 \%$. Les teneurs en $\mathrm{Mg}$ augmentent sensiblement en allant de la périphérie au le coeur du filon $(0.02 \%$ à $0.04 \%$ ) alors que dans les autres zones elles ne dépassent pas les $0.02 \%$. Nous observons la même chose pour le calcium $(0.03 \%$ à $0.06 \%)$ contre $0.03 \%$ au maximum dans les autres zones. Nous pouvons remarquer l'absence de potassium dans ces minéraux. Les teneurs en $\mathrm{Na}$ sont parfaitement corrélées à celles de $\mathrm{Zn}$.

Les minéraux du groupe de l'helvite séparés montrent de fortes teneurs en Sc (110 à 119 ppm), en Y (150 à 176 ppm), en Cu (165 à 273 ppm), en Nb (162 à 229 ppm) et en terres rares lourdes (56 à 81 ppm de $\mathrm{Yb}$ ). La somme des terres rares lourdes varie entre 102 et $145 \mathrm{ppm}$. Les teneurs en terres rares légères oscillent entre 1 et $3.5 \mathrm{ppm}$ de La et 10 à 13 ppm de Nd. Les teneurs en Ba sont faibles: 29 à 40 ppm. Les fortes teneurs en $\mathrm{Ba}$ et en Li correspondent à des faibles teneurs en $\mathrm{Be}$. Les teneurs en $\mathrm{Y}$ et en $\mathrm{Zn}$ sont corrélées négativement.

Des sulfures (pyrrhotite, chalcopyrite, sphalérite et galène) sont associés à l'helvite. Toutes les sphalérites du massif de Sucuri ont des teneurs en fer qui les situent dans le domaine de stabilité de la pyrite (Scott \& Barnes 1971) au voisinage de l'équilibre pyrrhotite-pyrite. Le comportement du fer dans la sphalérite ( 20 à $19 \%$ moles FeS dans la zone interne et $14-13 \%$ mole FeS dans la zone externe) et la présence de pyrrhotite précoce suggèrent que le système évolue à fugacité de soufre croissante et à température constante. Le cuivre entre dans le réseau de la sphalérite sous forme de solution solide $\mathrm{CuS}$, la modification de la solubilité du Fe qui en résulte est appréciée par le déplacement des points multiples en présence d'une phase riche en $\mathrm{Cu}$ (Wiggins \& Craig 1980). On obtient pour les deux groupes de sphalérites des températures de l'ordre de $525^{\circ} \mathrm{C}$ (pour une moyenne de $0.57 \%$ en poids de $\mathrm{Cu}$ ).

L'étude microthermométrique des inclusions fluides contenues dans la danalite a montré que les inclusions primaires (50 micromètres) sont biphasées acqueuses (la phase acqueuse représente $70 \%$ à $80 \%$ $\mathrm{du}$ volume total de l'inclusion) et de forme régulière. Ces inclusions fluides sont caractérisées par la présence de $\mathrm{CO}_{2}$, mise en évidence par formation de clathrate $\mathrm{CO}_{2} 53 / 4 \mathrm{H}_{2} \mathrm{O}$ lors du refroidissement. La fusion $\mathrm{du}$ clathrate complexe a été observé entre $-4^{\circ} \mathrm{C}$ et $-3.4^{\circ} \mathrm{C}$. La température de fin de fusion de la glace varie entre $-3.8^{\circ} \mathrm{C}$ et $-3^{\circ} \mathrm{C}$. D'après les données de Collins (1979) la salinité des inclusions dans le système $\mathrm{H}_{2} \mathrm{O}-\mathrm{CO}_{2}$ $\mathrm{NaCl}$ est de l'ordre de $20 \%$ en poids équivalent $\mathrm{NaCl}$. La pression partielle du $\mathrm{CO}_{2}$ dans les inclusions est de 35 bars. La température d'homogénéisation des inclusions est faible entre $170^{\circ} \mathrm{C}$ et $180^{\circ} \mathrm{C}$.

\section{Greisen}

Dans le greisen à muscovite qui résulte de la transformation du granite fin du massif de Sucuri, la danalite cristallise dans la zone de transformation de la biotite en muscovite. Le quartz (59\% de la composition modale de la roche) et la muscovite (38\%) dominent dans cette zone. L'albite est en faible 
quantité. La phénacite, l'helvite, fluorine et dans une moindre mesure la biotite, sont sporadiques. La cassitérite cristallise aux épontes. Dans certains cas elle est incluse dans la danalite.

La transformation de la biotite (sidérophyllite) en muscovite est contrôlée par les activités du fer et de la silice.

(1) $\mathrm{K}_{2} \mathrm{Fe}^{2+}{ }_{4} \mathrm{Al}_{2} \mathrm{Al}_{4} \mathrm{Si}_{4} \mathrm{O}_{20}(\mathrm{OH}, \mathrm{F})_{4}+2 \mathrm{SiO}_{2} \longrightarrow$ $\mathrm{K}_{2} \square{ }_{2} \mathrm{Al}_{4} \mathrm{Al}_{2} \mathrm{Si}_{6} \mathrm{O}_{2} \mathrm{O}(\mathrm{OH}, \mathrm{F})_{4}+4 \mathrm{FeO}$

$\square$ est le site octaédrique vacant.

Cette transformation peut être couplée avec la formation de la danalite qui fixe le fer si le soufre et le béryllium sont apportés par le fluide. L'absence de sulfure et la présence de la phénacite suggèrent que l'activité de soufre était faible. La limite de stabilité de la danalite peut être définie par l'équation:

(2) $8 \mathrm{Fe}_{3} \mathrm{O}_{4}+9 \mathrm{Be}_{2} \mathrm{SiO}_{4}+9 \mathrm{SiO}_{2}+3 \mathrm{~S}_{2} \rightarrow 3 \mathrm{Fe}_{8} \mathrm{Be}_{6} \mathrm{Si}_{6} \mathrm{O}_{24} \mathrm{~S}_{2}+7 \mathrm{O}_{2}$

Nous remarquons que la formation de la danalite est favorisée par un excès de silice, une forte fugacité du soufre et une faible fugacité d'oxygène. La présence de cassitérite en inclusion dans la danalite suggère que cet équilibre s'est déplacé vers la droite. En effet, la solubilité de la cassitérite augmente quand la fugacité d'oxygène diminue.

La danalite présente une zonation modeste, correspondant à une baisse du fer du coeur $\mathrm{Ge}_{24} \mathrm{Da}_{46} \mathrm{He}_{30}$ à la bordure $\mathrm{Ge}_{35} \mathrm{Da}_{42} \mathrm{He}_{23}$.

\section{Veines}

Les veines à sidérophyllite et les veines à quartzmuscovite sont localisées seulement dans le granite fin, au Nord du massif de Sucuri au garimpo Vaca Velha (Fig. 1). La succession paragénétique est la suivante:

1 - Dépôt dans les veines à sidérophyllites de: sidérophyllite, grenat $(61 \%$ spessartine et $37 \%$ almandin), phénacite, wolframite, quartz, fluorine et fluoroapatite.

2 - Dépôt dans les veines à quartz-muscovite de: muscovite, quartz, béryl et fluorine.

3 - Greisénisation de la veine à sidérophyllite et du granite fin et cristallisation de: muscovite, scheelite, columbite, cassitérite, fluoroapatite, sulfure et quartz.

4 - Albitisation des différentes veines et du greisen. Nous avons l'association: albite, helvite, phénacite et sulfure.

VEINE À SIDÉROPHYLLITE: La veine centimétrique est formée essentiellement de sidérophyllite ( $85 \%$ du volume). Dans les veines à sidérophyllite du massif de Serra Branca, le béryllium est généralement sous forme de béryl (Andrade \& Danni 1978) mais à Sucuri il est sous forme de phénacite associée au grenat. L'apparition du grenat (due à la richesse en $\mathrm{Mn}$ du fluide) a favorisé la cristallisation de la phénacite plutôt que celle du béryl. En effet, selon la réaction suivante:

$2 \mathrm{Be}_{3} \mathrm{Al}_{2} \mathrm{Si}_{6} \mathrm{O}_{18}+6 \mathrm{MnO} \rightarrow$

$2 \mathrm{Mn}_{3} \mathrm{Al}_{2} \mathrm{Si}_{3} \mathrm{O}_{12}+3 \mathrm{Be}_{2} \mathrm{SiO}_{4}+3 \mathrm{SiO}_{2}+6 \mathrm{O}_{2}$

une forte activité du $\mathrm{Mn}$ provoque la déstabilisation du béryl. Nous avons donc un contrôle géochimique

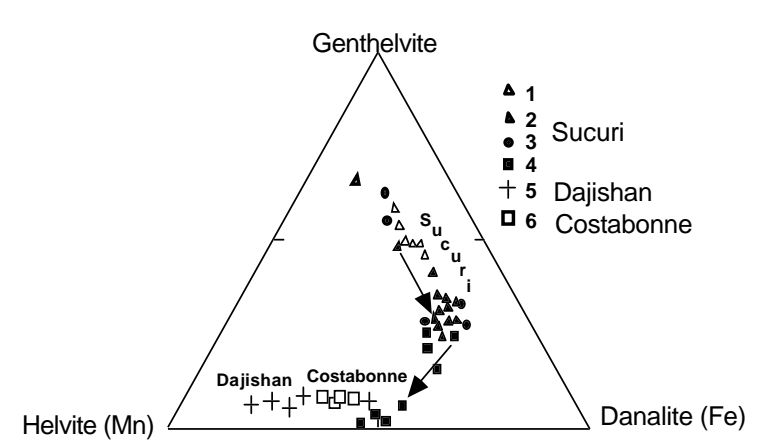

Figure 2: Composition des minéraux du groupe de l'helvite dans le diagramme molaire helvite $(\mathrm{Mn})$ : genthelvite $(\mathrm{Zn})$ - danalite $(\mathrm{Fe})$. Dans le massif de

Sucuri, nous avons les albitites: genthelvitesdanalites de la zone interne (1) et danalites de la zone externe (2); les veines à siderophyllite albitisées (3) et les veines à quartz-muscovite albitisées (4). La veine à quartz-muscovite (5) du massif de Dajishan (Chine) et les skarns (6) de Costabonne (France).

Figure 2: Mineral composition of helvite group within the ternary diagram: helvite $(\mathrm{Mn})$ genthelvite $(\mathrm{Zn})$ - danalite $(\mathrm{Fe})$.

de la cristallisation par le Mn.

Dans la partie albitisée de la veine à sidérophyllite, l'helvite cristallise aux dépens du grenat et de la phénacite (suivant la réaction):

$8 \mathrm{Mn}_{3} \mathrm{Al}_{2} \mathrm{Si}_{3} \mathrm{O}_{12}+9 \mathrm{Be}_{2} \mathrm{SiO}_{4}+8 \mathrm{Na}_{2} \mathrm{O}+33 \mathrm{SiO}_{2}+3 \mathrm{~S}_{2} \rightarrow$ $3 \mathrm{Mn}_{8} \mathrm{Be}_{6} \mathrm{Si}_{6} \mathrm{O}_{24} \mathrm{~S}_{2}+16 \mathrm{NaAlSi}_{3} \mathrm{O}_{8}+\mathbf{3 O}_{2}$

La formation de l'helvite est contrôlée essentiellement, toutes choses égales par ailleurs, par l'activité du soufre en solution.

L'helvite présente une composition de danalite $\mathrm{Ge}_{25} \mathrm{Da}_{42} \mathrm{He}_{33}$ dans la zone externe faiblement albitisée et une composition de genthelvite $\left(\mathrm{Ge}_{41} \mathrm{Da}_{39} \mathrm{He}_{20}\right.$ à $\mathrm{Ge}_{55} \mathrm{Da}_{39} \mathrm{He}_{22}$ ) dans la zone interne fortement albitisée (Fig. 2). Certaines helvites sont zonées. Elles montrent un coeur plus riche en $\mathrm{Mn}, \mathrm{Zn}$ et $\mathrm{Na}$ et plus pauvre en Fe que la bordure.

VEINE À QUARTZ ET MUSCOVITE: Cette veine à quartz-muscovite est centimétrique et elle recoupe la veine à sidérophyllite qu'elle renferme aussi en enclave. Dans cette veine, la paragenèse primaire est à quartz, muscovite, béryl, avec ou sans sidérophyllite. Le béryl et la sidérophyllite sont muscovitisés (réaction (5)) et albitisés (réaction (6)). (5) $6 \mathrm{Be}_{3} \mathrm{Al}_{2} \mathrm{Si}_{6} \mathrm{O}_{18}+6 \mathrm{H}_{2} \mathrm{O}+4 \mathrm{~K}^{+} \rightarrow$ $4 \mathrm{H}^{+}+4 \mathrm{KAl}_{2}\left(\mathrm{AlSi}_{3} \mathrm{O}_{10}\right)(\mathrm{OH})_{2}+9 \mathrm{Be}_{2} \mathrm{SiO}_{4}+15 \mathrm{SiO}_{2}$ (6) $2 \mathrm{Be}_{3} \mathrm{Al}_{2} \mathrm{Si}_{6} \mathrm{O}_{18}+3 \mathrm{SiO}_{2}+2 \mathrm{H}_{2} \mathrm{O}+4 \mathrm{Na}^{+} \rightarrow$

$4 \mathrm{H}^{+}+4 \mathrm{NaAISi}_{3} \mathrm{O}_{8}+3 \mathrm{Be}_{2} \mathrm{SiO}_{4}$

Trois types d'associations ont été observés dans cette veine:

1 - Dans les enclaves de l'ancienne veine à sidérophyllite, nous avons la même situation que dans la veine à sidérophyllite, l'helvite $\mathrm{Ge}_{1} \mathrm{Da}_{38} \mathrm{He}_{61}$ se développe sur le grenat et la phénacite.

2 - Dans la veine à quartz et muscovite (en dehors 
des enclaves) la danalite $\mathrm{Ge}_{17} \mathrm{Da}_{54} \mathrm{He}_{29}$ englobe les sidérophyllites et la phénacite.

3 - Dans les zones à veinule d'albite, nous avons l'association quartz, muscovite et albite. La teneur du pôle genthelvite de la danalite augmente de $\mathrm{Ge}_{20}$ $\mathrm{Da}_{52} \mathrm{He}_{27}$ à $\mathrm{Ge}_{39} \mathrm{Da}_{34} \mathrm{He}_{27}$.

\section{Gisement de Dajishan, (CHINE)}

VEINE À QUARTZ ET MUSCOVITE: Le gisement à wolframite de Dajishan a été étudié en détail par Fonteilles et al. (1987, 1990) et par Raimbault et al. (1987) ce qui nous a permis d'aborder les relations entre les minéraux de béryllium (Fig. 3).

\section{Skarns (skarn Nord de Costabonne)}

L'helvite est un minéral assez fréquent dans les skarns. Elle a été rencontrée en France dans les skarns de Costabonne (Guitard \& Laffite 1958) et dans ceux de Loudenvielle, développés sur des niveaux carbonatés (Ragu 1994), aux Etats Unis dans les skarns de New Mexico (Glass et al. 1944) et au Maroc dans les skarns de Azegour (Walenta 1981).

Dans la zone axiale des Pyrénées et à $50 \mathrm{kms}$ environ de la terminaison orientale de la chaine, un gîte de skarn se trouve au contact du granite de Costabonne circonscrit par des calcaires et dolomies d'âge cambrien (Fig. 4). Ces skarns forment plusieurs

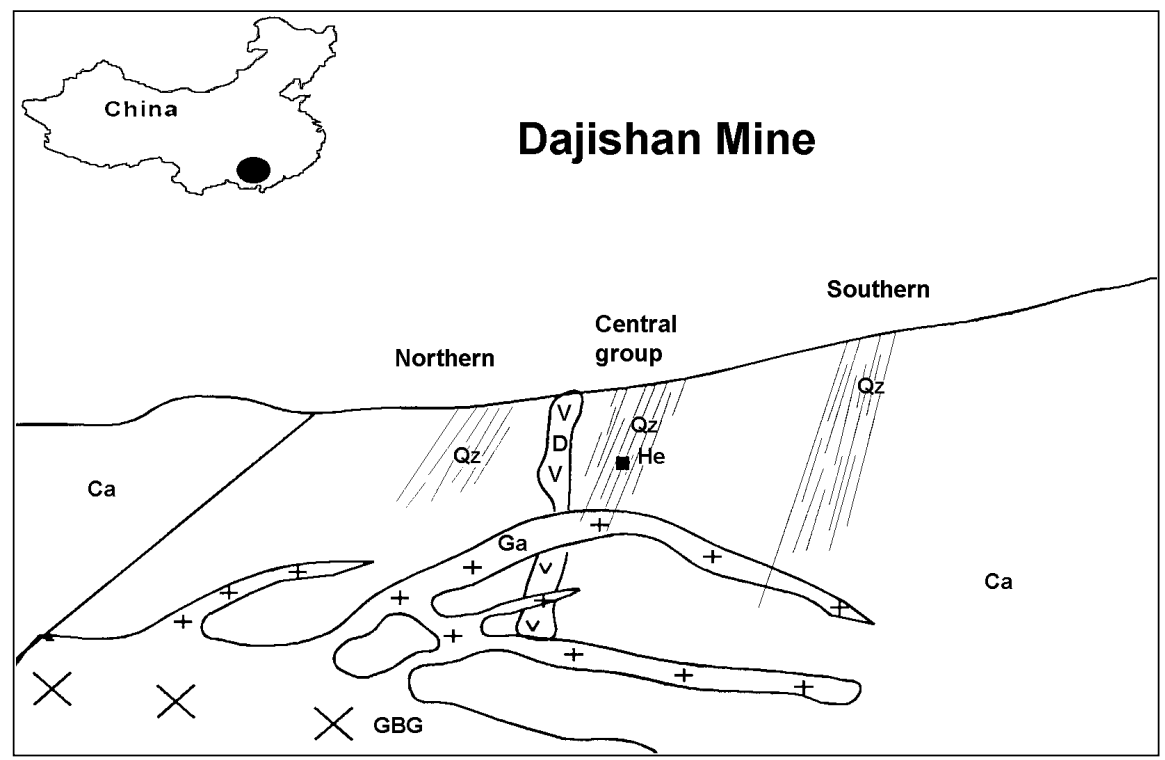

Figure 3: Coupe schématique du gisement de Dajishan (Jiangxi, China). GBG - granite à biotite; GA - granite à albite; D - dyke de diorite; Ca - métasédiment cambrien; Qz - veines à quartz-wolframite et He - localisation des échantillons de l'helvite étudiés.

Figure 3:Cross-section trough the Dajishan tungsten deposit (Jiangxi, China). CBG - biotite granite; $G A$ - garnet bearing albite granite; $D$ - diorite dyke; $C a$ - cambrian strata; $Q z$ - quartz-wolframite veins and He - location of the samples of helvite analysed.

$\mathrm{Au}$ point de vue minéralogique, nous observons une évolution de la composition du grenat et de la muscovite dans les veines minéralisées. Il s'agit d'un enrichissement progressif: - en $\mathrm{MgO}: 0.08 \%$ à $0.19 \%$ dans le grenat, $0.3 \%$ à $0.75 \%$ dans les muscovites seules et $0.9 \%$ à $2.1 \%$ dans les muscovites associées aux biotites tardives; - en $\mathrm{CaO}$ pour les grenats $2.4 \%$ à $16.8 \%$ du stade II. Finalement nous aboutissons au développement limite de biotite et de feldspath sur la muscovite et apparemment aux dépens de ce minéral.

Le béryl est associé à la muscovite, au quartz et à la fluorine (la paragenèse primaire). La cristallisation de l'helvite coïncide avec le stade II de la transformation de la wolframite en scheelite II et au développement du grenat plus calcique à la périphérie des cristaux précédemment formés. La formation de l'helvite $\mathrm{Ge}_{10} \mathrm{Da}_{30} \mathrm{He}_{60}$ est favorisée (réaction (7)) par l'augmentation de l'activité de $\mathrm{Ca}$ et de $\mathrm{Mn}$ dans le fluide.

(7) $2 \mathrm{Be}_{3} \mathrm{Al}_{2} \mathrm{Si}_{3} \mathrm{O}_{18}+6 \mathrm{CaO}+8 \mathrm{MnO}+6 \mathrm{SiO}_{2}+\mathrm{S}_{2} \longrightarrow$ $2 \mathrm{Ca}_{3} \mathrm{Al}_{2} \mathrm{Si}_{3} \mathrm{O}_{12}+\mathrm{Mn}_{8} \mathrm{Be}_{6} \mathrm{Si}_{6} \mathrm{O}_{24} \mathrm{~S}_{2}+\mathrm{O}_{2}$ grands corps subconcordants dont les plus importants sont situés à la base des dolomies, au contact du granite (skarn Nord) et à leur toit sous les micaschistes (skarn Sud).

L'helvite a été trouvée par Guitard \& Laffite (1958) dans la zone de pyroxénite claire des skarns Nord. Dubru (1986) décrit des minéraux de béryllium (musgravite et broméllite) dans les veines à silicates et spinelle du marbre dolomitique (skarn à brucite).

Dans la roche à helvite, l'amphibole verte, fréquemment associée à la calcite, se développe aux dépens des pyroxènes primaires. La composition de cette amphibole est caractérisée par un rapport $\mathrm{Mg}$ / $(\mathrm{Fe}+\mathrm{Mg})$ variant de 0.95 à 0.98 et des teneurs en $\mathrm{MnO}$ de $0.25 \%$. Elle est accompagnée de quartz. La pyrite et la sphalérite se développent de façon relativement abondante. Cette zone de skarn est caractérisée par de faibles teneurs en Mn. L'helvite, de composition $\left(\mathrm{Ge}_{9} \mathrm{Da}_{36} \mathrm{He}_{55}\right)$, est le seul minéral manganésifère.

Fonteilles et al. (1980) ont estimé la température de la phase secondaire à laquelle appartient l'helvite 

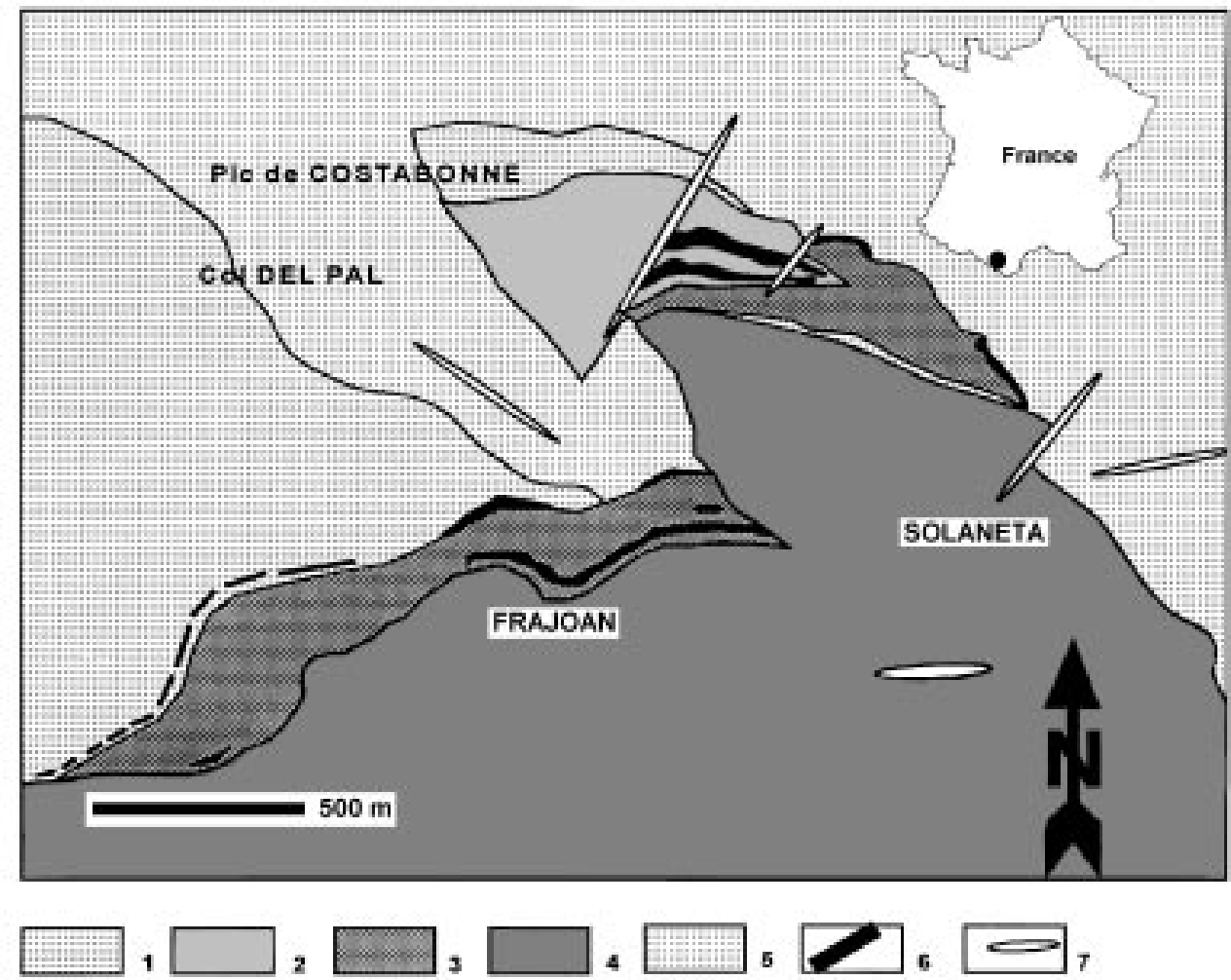

Figure 4: Carte géologique du massif de Costabonne (France) d'après Guitard \& Laffite (1958). 1 - gneiss; 2 - cornéenne; 3 - calcaire; 4 - micaschiste; 5 - granite et 6 - occurrence de skarn.

Figure 4: Geological map of the Costabonne massif (France) from Guitard \& Laffite (1958). 1 - gneisses; 2 - hornfels; 3 - limestone; 4 - micaschist; 5 - granite; 6 - skarn occurrences.

entre $350^{\circ} \mathrm{C}$ et $450^{\circ} \mathrm{C}$ pour des pression de l'ordre de 1.5 à 2 kb. L'étude microthermométrique (Guy 1988) sur les inclusions fluides dans les quartz hydrothermaux montre que la salinité du fluide est variable de $13 \%$ à $25 \%$ en poids équivalent $\mathrm{NaCl}$. Les conditions de fugacité de soufre sont celles du domaine de stabilité de la pyrite, à proximité du domaine de la pyrrhotite.

\section{DISCUSSION}

\section{Composition}

Des teneurs en $\mathrm{Na}$ ont été obtenues dans différents minéraux du groupe de l'helvite. Elles sont parfaitement corrélées à celles de $\mathrm{Zn}$. Une faible proportion de $\mathrm{Na}$ entre dans la structure de l'helvite.

Pour tenter de comprendre les mécanismes d'introduction de Na dans la structure de l'helvite, il nous est apparu nécessaire de procéder à une étude expérimentale sur un système simple. Nous avons réalisé deux séries d'expériences à $450^{\circ} \mathrm{C}$ et à $600^{\circ} \mathrm{C}$ et à $1.5 \mathrm{~kb}$ avec des solutions à différentes concentrations en $\mathrm{NaCl}$. Il serait prématuré d'interpréter dans le détail ces expériences qui sont encore en cours. On retiendra cependant que les analyses à la microsonde de la genthelvite synthétisée semblent montrer qu'une proportion de $\mathrm{Na}$ entre effectivement dans le réseau de l'helvite et qu'une faible proportion de soufre s'échange avec le $\mathrm{Cl}$.
L'helvite de formule (Fe, $\mathrm{Zn}, \mathrm{Na}, \mathrm{Mn})_{8} \mathrm{Be}_{6}\left(\mathrm{SiO}_{4}\right)_{6} \mathrm{~S}_{2}$, cristallise dans le système cubique. Elle appartient au groupe d'espace P $\overline{4}$ 3n (Hassan \& Grundy 1985)).

Dans la structure de la sodalite nous avons une organisation de $\mathrm{Cl}$ qui est cubique centré (chaque $\mathrm{Cl}$ est un des centres des "cages" définies par les tétraèdres $\mathrm{Al}$ et $\mathrm{Si}$ ). Les ions $\mathrm{Na}$ sont eux aussi parfaitement localisés: quatre $\mathrm{Na}$ autour de chaque $\mathrm{Cl}$. Nous savons que ces sites $\mathrm{Cl}$ et $\mathrm{Na}$ peuvent ne pas être complètement occupés: par exemple dans la haüyne $(\mathrm{Na}, \mathrm{Ca})_{8-4} \mathrm{Al}_{6} \mathrm{Si}_{6} \mathrm{O}_{24}\left(\mathrm{SO}_{4}\right)_{1-2}$ (Barth 1926). D'après les études de Hassan \& Grundy (1985) le nombre de cations dans le site octaédrique de l'helvite par formule unité ne doit pas dépasser 8 . Donc nous ne pouvons pas remplacer $\mathrm{Fe}^{2+}$ ou $\mathrm{Mn}^{2+}$ par $2 \mathrm{Na}^{+}$. Compte-tenu de ce qui précède et par analogie à la haüyne, la formule structurale de l'helvite pourrait s'écrire:

\section{$(\mathrm{Mn}, \mathrm{Zn}, \mathrm{Fe})_{8-\mathrm{x}} \mathrm{Na}_{x} \mathrm{Be}_{6} \mathrm{Si}_{6} \mathrm{O}_{24} \mathrm{~S}_{2-\mathrm{x}} \mathrm{Cl}_{x}$}

où $\mathrm{x}$ est la fraction atomique de $\mathrm{Na}$ qui entre dans la structure. Une partie de S est remplacée par $\mathrm{Cl}$.

Dans les danalites séparées du massif de Sucuri les teneurs en Li (19 à 49 ppm) sont corrélées négativement avec celles en Be (13.39\% à $13.04 \%$ de $\mathrm{BeO})$. Le Be est toujours inférieur à Si dans la formule structurale calculée sur la base de 24 oxygènes. Le Li remplace probablement le Be dans la structure de l'helvite et le déficit de charge ainsi créé est compensé par l'introduction de $\mathrm{Na}$ dans la structure. 


\begin{tabular}{cccccccccccccc}
\hline Número & 1 & 2 & 3 & 4 & 5 & 6 & 7 & 8 & 9 & 10 & 11 & 12 \\
$\mathrm{Tipo}$ & $\mathrm{SDA}$ & $\mathrm{SDA}$ & $\mathrm{QMI}$ & $\mathrm{GMI}$ & $\mathrm{GME}$ & $\mathrm{QME}$ & $\mathrm{GA} 1$ & $\mathrm{GA2}$ & $\mathrm{AB}$ & $\mathrm{AB}$ & $\mathrm{SK}$ & $\mathrm{QM}$ \\
\hline $\mathrm{SiO}_{2}$ & 31,97 & 31,81 & 32,7 & 32,73 & 32,9 & 32,9 & 32,6 & 32,85 & 33,28 & 32,92 & 32,57 & 32,23 \\
$\mathrm{BeO}^{*}$ & 13,17 & 13,16 & 13,05 & 12,97 & 12,85 & 13,01 & 13,08 & 13,07 & 12,87 & 12,92 & 13,12 & 12,97 \\
$\mathrm{Al}_{2} \mathrm{O}_{3}$ & 0,03 & 0,06 & 0,04 & n.d. & 0,1 & 0,02 & 0,04 & n.d. & 0,08 & 0,01 & 0,03 & 0,07 \\
$\mathrm{FeO}^{* *}$ & 19,06 & 11,19 & 23,07 & 27,71 & 22,2 & 23,2 & 24,33 & 23,3 & 12018 & 495 & 18,21 & 13,9 \\
$\mathrm{MnO}$ & 9,88 & 10,64 & 15,08 & 16,03 & 2810 & 27,9 & 11,12 & 9,66 & 9,28 & 12,13 & 27,93 & 31,88 \\
$\mathrm{ZnO}$ & 23,13 & 30,24 & 13,9 & 7,6 & 0,09 & 0,16 & 15,83 & 18,52 & 31,01 & 35,53 & 5,12 & 4,93 \\
$\mathrm{MgO}$ & 0,02 & n.d. & n.d. & n.d. & n.d. & n.d. & 0,02 & n.d. & 0,04 & n.d. & 0,12 & 0,06 \\
$\mathrm{CaO}$ & 0,05 & 0,1 & 0,01 & 0,02 & 0,01 & n.d. & 0,03 & 0,02 & 0,06 & 0,04 & 0,06 & 0,03 \\
$\mathrm{Na} \mathrm{O}_{2} \mathrm{O}$ & 0,22 & 0,26 & 0,19 & 0,07 & 0 & 0,02 & 0,15 & 0,18 & 0,29 & 0,31 & 0,21 & 0,21 \\
$\mathrm{~K}{ }_{2} \mathrm{O}$ & 0,01 & 0,01 & 0,01 & 0,01 & & & & & & & & \\
$\mathrm{~S}$ & 5,46 & 5,42 & 5,78 & 5,78 & 5,43 & 5,53 & 5,69 & 5,54 & 5,54 & 5,51 & 5,66 & 5,66 \\
\hline Total & 103,07 & 102,95 & 103,84 & 102,93 & 101,82 & 102,75 & 103,08 & 102,8 & 103,61 & 103,35 & 103,03 & 102,84 \\
$\mathrm{O}=\mathrm{S}$ & 2,72 & 2,7 & 2,88 & 2,88 & 2,71 & 2,76 & 2,84 & 2,76 & 2,76 & 2,75 & 2,82 & 12,82 \\
\hline Total & 100,35 & 100,25 & 100,96 & 100,05 & 99,11 & 99,99 & 100,24 & 100,04 & 100,85 & 100,6 & 100,21 & 100,84 \\
\hline
\end{tabular}

\section{Formule structurale sur la base de $26(0,5)$}

\begin{tabular}{ccccccccccccc}
\hline $\mathrm{Be}$ & 6,02 & 6,02 & 5,97 & 5,93 & 5,88 & 5,95 & 5,98 & 5,98 & 5,88 & 5,91 & 6 & 5,99 \\
$\mathrm{Si}$ & 6,04 & 6,06 & 6,05 & 6,06 & 6,06 & 6,04 & 6,09 & 6,07 & 6,14 & 6,15 & 6,01 & 6,09 \\
$\mathrm{Al}$ & 0,01 & 0,01 & 0,01 & 0 & 0,02 & 0 & 0,01 & 0 & 0,02 & 0 & 0,01 & 0,02 \\
& 12,07 & 12,09 & 12,03 & 11,99 & 11,96 & 11,99 & 12,08 & 12,05 & 12,04 & 12,06 & 12,02 & 11,99 \\
\hline $\mathrm{Fe}$ & 3,01 & 1,78 & 3,57 & 4,29 & 3,42 & 3,56 & 3,8 & 3,74 & 1,88 & 0,77 & 2,81 & 2,13 \\
$\mathrm{Mn}$ & 1,58 & 1,72 & 2,36 & 2,51 & 4,39 & 4,34 & 1,76 & 1,51 & 1,45 & 1,92 & 4,37 & 4,95 \\
$\mathrm{Zn}$ & 3,23 & 4,26 & 1,9 & 1,04 & 0,02 & 0,02 & 2,19 & 2,53 & 4,23 & 4,91 & 0,7 & 0,67 \\
$\mathrm{Mg}$ & 0 & 0 & 0 & 0 & 0,01 & 0 & 0,01 & 0 & 0,03 & 0,02 & 0 & 0 \\
$\mathrm{Ca}$ & 0,01 & 0,02 & 0 & 0 & 0 & 0 & 0,01 & 0 & 0,01 & 0,01 & 0,01 & 0,01 \\
$\mathrm{Na}$ & 0,08 & 0,09 & 0,05 & 0,03 & 0 & 0 & 0,05 & 0,06 & 0,1 & 0,11 & 0,08 & 0,07 \\
$\mathrm{~K}$ & 0 & 0 & 0 & 0 & & & & & & & & \\
& 83 & 8,02 & 7,95 & 7,9 & 7,84 & 7,9 & 7,91 & 7,94 & 7,85 & 7,87 & 7,99 & 7,85 \\
\hline $\mathrm{S}$ & 1,93 & 1,93 & 2 & 2,01 & 2,01 & 1,9 & 1,92 & 1,981 & 1,92 & 1,93 & 1,84 & 1,83 \\
\hline
\end{tabular}

* Les teneurs en Be ont été calculées selon la méthode de Dunn (1976): $(\mathrm{Mn}+\mathrm{Fe}+\mathrm{Zn}+\mathrm{Ca}+\mathrm{Mg}+\mathrm{Na}) / \mathrm{Be}=4 / 3$. ** tout le Fe est sous la forme de $\mathrm{FeO}$

SDA: veine de sidérophyllite albitisée; QMI : partie interne de la veine de quartz-muscovite fortemente albitisée; QME: partie externe de la veine de quartz-muscovite fortemente albitisée; GA: granite albitisé zone 1 et zone 2 et AB: albitite (zone 3) du massif de Sucuri; SK: skarn de Costabonne (France) et QM: veine de quartz-moscovite de Dajishan (Chine).

Tableau 2 : Composition représentative des minéraux du groupe de l'helvite du massif de Sucuri, des skarns de Costabonne et du massif de Dajishan.

Table 2: Representative composition of helvite group minerals from the Sucuri massive, skarns from 


\begin{tabular}{|c|c|c|c|c|c|c|c|c|c|}
\hline Localit & & Sucuri & & & & Daji & shan & & \\
\hline Sample & Su3 & SU3 & SU3 & $\begin{array}{l}517- \\
36 / \mathrm{A}\end{array}$ & $\begin{array}{l}517- \\
36 / \mathrm{B}\end{array}$ & $\begin{array}{l}517- \\
39 / A\end{array}$ & $\begin{array}{l}517- \\
39 / \mathrm{B}\end{array}$ & $\begin{array}{l}517- \\
39 / \mathrm{A}\end{array}$ & $\begin{array}{l}517- \\
39 / \mathrm{B}\end{array}$ \\
\hline ppm & & & & & & & & & \\
\hline Sc & 106 & 119 & 118 & 6,84 & 7,36 & 8,58 & 9,63 & 8,17 & 8,51 \\
\hline $\mathrm{Cr}$ & $<8$ & $<6$ & $<8$ & na & $<2$ & na & $<3$ & na & $<2$ \\
\hline Co & 12,3 & 14,2 & 9,8 & 2,45 & 2,73 & 2,18 & 2,4 & 2,13 & 2,44 \\
\hline $\mathrm{Ga}$ & $<21$ & $<13$ & na & na & 9 & na & $<14$ & na & 17 \\
\hline $\mathrm{Se}$ & 8 & 5 & 8 & 1,2 & 2,5 & 0,2 & 0,7 & 0,6 & \\
\hline $\mathrm{Ag}$ & 18 & 5 & 8 & 1,2 & 2,5 & 0,2 & 0,7 & 0,6 & 1 \\
\hline $\mathrm{Cd}$ & $<100$ & $<100$ & $<110$ & 226 & 175 & 203 & 174 & 145 & 171 \\
\hline $\mathrm{Sb}$ & $<1.2$ & 2,3 & $<1,4$ & 3,1 & 4,8 & 0,51 & 0,82 & 0,26 & 0,52 \\
\hline $\mathrm{Ta}$ & $<1,6$ & 1,4 & 4,5 & 0,58 & 7,5 & 0,42 & 0,35 & 0,49 & 0,52 \\
\hline W & $<30$ & $<18$ & na & 2880 & 1344 & na & $<9$ & na & $<7$ \\
\hline Auppb & $<23$ & $<24$ & $<25$ & 24 & 9 & na & $<9$ & na & $<7$ \\
\hline Th & 3,4 & 2,4 & 26,8 & $<0,2$ & 0,2 & $<0,2$ & $<0,3$ & $<0,2$ & 0,13 \\
\hline $\mathrm{La}$ & 2,4 & 0,9 & 10,4 & 1,21 & 1,01 & 0,6 & 0,81 & 0,63 & 0,63 \\
\hline $\mathrm{Ce}$ & 5 & 4,2 & 53 & 2,3 & 2 & 1,4 & 2,6 & 1,3 & 1,9 \\
\hline Sm & 3,32 & 3,93 & 34,6 & 0,28 & 0,31 & 0,37 & 0,46 & 0,37 & 0,54 \\
\hline $\mathrm{Eu}$ & 0,14 & 0,13 & 1,27 & 0,3 & 0,21 & 0,27 & 0,36 & 0,32 & 0,54 \\
\hline $\mathrm{Gd}$ & nd & nd & 63,5 & nd & nd & na & nd & na & nd \\
\hline $\mathrm{Tb}$ & 1,8 & 1,9 & 12,4 & 0,43 & nd & 0,67 & 0,63 & 0,5 & 0,4 \\
\hline $\mathrm{Yb}$ & 57,4 & 63,2 & 146 & 7,35 & 8,14 & 8,16 & 9,35 & 7,41 & 7,88 \\
\hline $\mathrm{Lu}$ & 19,7 & 22,6 & 42 & $\mid 1,81$ & 1,86 & 1,65 & 1,94 & 1,55 & 1,66 \\
\hline
\end{tabular}

Tableau 3: Analyses des minéraux du groupe de l'helvite par activation neutronique. Table 3: Analysis of helvite group minerals by neutron activation.

Une simple revue des analyses dans le tableau 3 montre que le $\mathrm{Ta}, \mathrm{Ag}, \mathrm{Sb}$ et $\mathrm{Au}$ ne se trouvent probablement pas dans le réseau de l'helvite. Par contre le $\mathrm{Sc}, \mathrm{Co}, \mathrm{Cd}$, Y et les terres rares peuvent être incorporés dans l'helvite en substitution à Fe, Mn, Zn. Le Se est en situation intermédiaire et peut remplacer l'anion S, mais les fortes variations de ses teneurs et celles des éléments de nature chalcophylle $\mathrm{Ag}, \mathrm{Sb}$ et Au suggèrent la présence de microinclusions de sulfure possédant du Se.

Le Sc et Co ont la même configuration électronique $3 \mathrm{dTE}$ que les cations $\mathrm{Mn}, \mathrm{Fe}, \mathrm{Zn}$. Les rayons ioniques $\mathrm{du} \mathrm{Co}^{2+}$ et $\mathrm{Sc}^{3+}$ sont respectivement $0.72 \AA$ et $0.73 \AA$ (Arhens 1952). Ils sont proches de ceux du $\mathrm{Fe}^{2+}(0.75$ $\AA)$ et de $\mathrm{Zn}^{2+}(0.74 \AA)$ mais différents de $\mathrm{Mn}^{2+}(0.8$ $\AA$ ). Les teneurs en Sc dans les helvites (Sucuri et Dajishan) varient très peu dans chaque occurence mais elles sont largement dispersées entre les deux gisements (108 à 119 ppm dans le massif de Sucuri et
7 à 9 ppm dans le massif de Dajishan). De même pour le Co (10 à 13 ppm à Sucuri et 2 à 2.5 ppm à Dajishan); Ces faibles teneurs en Co sont dues probablement à la faible abondance du Co dans ces environnements granitiques. Le relatif enrichissement en Sc dans les helvites de Sucuri pourrait être lié à la différence du fluide minéralisateur. Les teneurs en $\mathrm{Sc}$ semblent être liées à la composition de l'helvite $\left(\mathrm{Ge}_{29} \mathrm{Da}_{50} \mathrm{He}_{21}\right.$ à Sucucri et $\mathrm{Ge}_{9} \mathrm{Da}_{36} \mathrm{He}_{55}$ à Dajishan). Le Sc peut remplacer $\mathrm{Fe}$ ou $\mathrm{Zn}$ plus facilement que $\mathrm{Mn}$ dans la structure de l'helvite. Les contraintes cristallochimiques régissent l'introduction du Sc ( et dans une moindre mesure celle du Co) dans la structure de l'helvite.

La géochimie du $\mathrm{Cd}$ est étroitement liée à celle du Zn (Vlasov 1966). Le rayon ionique du $\mathrm{Cd}^{2+}(0.97 \AA)$ est cependant plus élevé que celui de $\mathrm{Zn}$ et plus proche de celui de Mn. Dans nos exemples, les helvites de Dajishan concentrent plus facilement le Cd (172 ppm) 
que les helvites de Sucuri (100 ppm). Le comportement du $\mathrm{Cd}^{2+}$ est à l'opposé de celui du $\mathrm{Zn}$ et du $\mathrm{Na}(0.17$ a./f.u. de Na à Sucuri et 0.068 a./f.u. de Na à Dajishan). $\mathrm{Na}$ a le même rayon ionique $0.97 \AA$ que celui de $\mathrm{Cd}$. Le facteur cristallochimique ne peut donc à lui seul expliquer l'introduction de $\mathrm{Cd}$ dans la structure de l'helvite. Et d'autres facteurs peuvent être envisagés, notamment le rapport $\mathrm{Zn} / \mathrm{Cd}$ dans le fluide et la salinité $\mathrm{du}$ milieu puisqu'une forte concentration de $\mathrm{Cl}$ augmente la solubilité du Cd par rapport à celle de $\mathrm{Zn}$ (Mookherjee 1962).

Les helvites montrent un enrichissement en terres rares lourdes par rapport aux terres rares légères. Le rapport $(\mathrm{La} / \mathrm{Yb})_{\mathrm{n}}$ normalisé aux chondrites est de l'ordre de 0.009 à 0.050 à Sucuri et de 0.05 à 0.11 à Dajishan. Dans un environnement franchement alcalin (albitite de Sucuri - Fig. 5) l'helvite concentre plus facilement les terres rares lourdes.

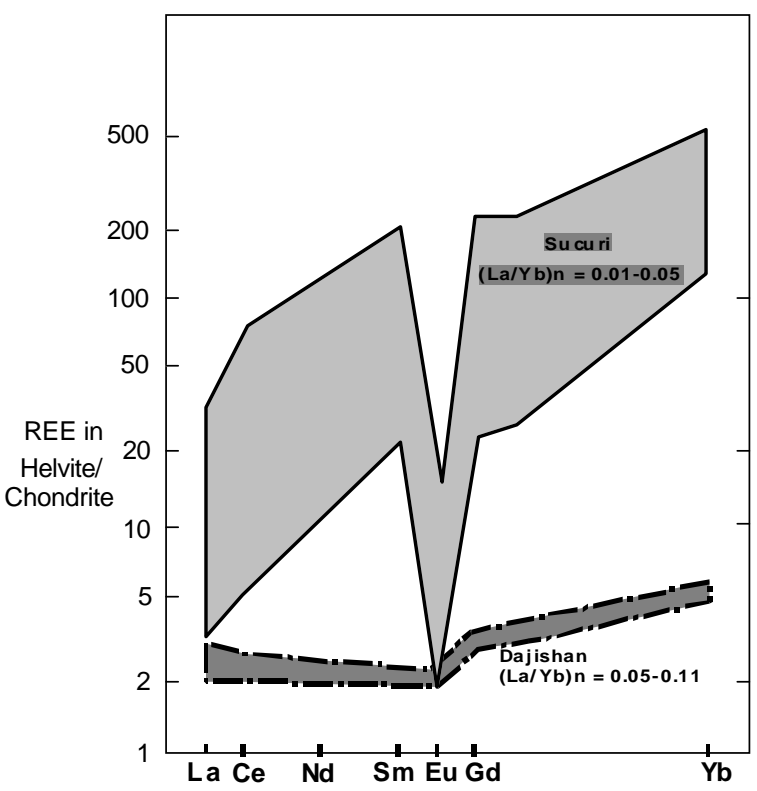

Figure 5: Normalisation des minéraux du groupe de l'helvite par rapport aux chondrites. Danalite dans les albitites du massif de Sucuri et helvite dans les veines à quartz-wolframite du massif de Dajishan.

Figure 5: REE patterns of the helvite group minerals; danalite of albitites from Sucuri massive and helvite of quartz wolframite veins from the Dajishan massive.

Les terres rares peuvent être complexées par les anions $\mathrm{F}^{-}, \mathrm{P}, \mathrm{Cl}^{-}$ou $\mathrm{Co}_{3}^{2-}$ (Mineyev 1963). La présence de fluorine dans les albitites de Sucuri et dans les veines à quartz et muscovite de Dajishan suggère que l'anion $\mathrm{F}^{-}$est le principal complexant des terres rares lourdes. Cependant, la présence d'une faible quantité de $\mathrm{CO}_{2}$ (mise en évidence par formation de clathrate) dans les inclusions de fluides dans l'helvite laisse penser que l'anion $\mathrm{CO}_{3}^{2-}$ a eu un rôle (mineur sans doute) dans le transport des terres rares. En effet, Dumonceau et al. (1979) ont montré que le transport par les ions tétracarbonatolanthanidates n'est possible que dans des conditions alcalines.

Les proportions du fluide en terres rares durant le stade II à Dajishan peuvent être estimées en utilisant la scheelite. D'après les données de Raimbault et al. (1987) le rapport $(\mathrm{La} / \mathrm{Yb})_{\mathrm{N}}$ du fluide peut être estimé grossièrement de 22 à 4.8. Nous pouvons calculer ce rapport dans le fluide hydrothermal en équilibre avec le granite fin en utilisant les données expérimentales de Flynn \& Burnham (1978). Nous trouvons une valeur de 18 pour le fluide. Vu la dispertion des résultats, nous ne pouvons pas expliquer la distribution observée des terres rares dans l'helvite par une forte concentration en terres rares lourdes des fluides hydrothermaux. Fort de ces considérations, nous pouvons conclure que les contraintes cristallochimiques jouent un rôle important dans l'introduction des terres rares dans la structure de l'helvite. Les cations de terres rares remplacent les cations majeurs $\mathrm{Fe}, \mathrm{Mn}, \mathrm{Zn}$. Ceci est bien connu dans la structure du grenat (comme de la wolframite) qui s'accommode plus facilement des terres rares lourdes que des terres rares légères. Ceci est vrai dans les conditions magmatiques (Irving \& Frey 1978) mais aussi dans les environnements hydrothermaux (Raimbault, en préparation). Nous proposons que l'incorporation des terres rares dans la structure de l'helvite obéit aux mêmes lois qui sont principalement régies par les variations de rayons ioniques. Dans le site octaédrique $\mathrm{Fe}^{2+}, \mathrm{Mn}^{2+}$ et $\mathrm{Zn}^{2+}$ ont respectivement des Ri $0.75 \AA, 0.8 \AA$ et $0.73 \AA$ (Arhens 1952) et pour les terres rares trivalentes le Ri varie entre $1.05 \AA$ pour La et $0.73 \AA$ pour Lu.

A partir de l'estimation du coefficient de fractionnement de La et $\mathrm{Yb}$ dans le fluide à Dajishan, nous pouvons évaluer le partage du rapport $\mathrm{La} / \mathrm{Yb}$ dans l'helvite et dans le fluide. La concentration de $\mathrm{Yb}$ par rapport à celle du La est 100 à 200 fois plus grande dans l'helvite que dans le fluide. A Sucuri le Yb est 200 à 500 fois plus concentré que le La dans l'helvite. La danalite $\mathrm{Ge}_{29} \mathrm{Da}_{50} \mathrm{He}_{21}$ (Sucuri) renferment deux fois plus de terres rares que l'helvite $\mathrm{Ge}_{9} \mathrm{Da}_{36} \mathrm{He}_{55}$ (Dajishan). Cependant, nous n'avons pas assez de données pour conclure à l'influence des substitutions $\mathrm{Mn}-\mathrm{Fe}-\mathrm{Zn}$ sur l'incorporation des terres rares. A Sucuri, le fluide franchement alcalin (albitite) a probablement favorisé l'introduction des terres rares dans l'helvite.

\section{Conditions physico-chimiques de formation}

FUGACITÉS DE SOUFRE ET D'OXYGÈNE: Examinons les domaines de stabilité de l'helvite par rapport à celui de la phénacite pour divers environnements minéralogiques à $500^{\circ} \mathrm{C}$. Nous avons utilisé les données thermodynamiques de Fursenko (1989) pour les minéraux de l'helvite et celles de Robie et al. (1979) pour les autres minéraux. Nos résultats à $50{ }^{\circ} \mathrm{C}$ sont différents de ceux de Fursenko (1989).

Le domaine de la stabilité de l'helvite est délimité par les équations:

(8) $8 \mathrm{MnS}+3 \mathrm{Be}_{2} \mathrm{SiO}_{4}+3 \mathrm{SiO}_{2}+3 \mathrm{O}_{2} \rightarrow \mathrm{Mn}_{8} \mathrm{Be}_{6} \mathrm{Si}_{6} \mathrm{O}_{24} \mathrm{~S}_{2}+3 \mathrm{~S}_{2}$ $\log f\left(O_{2}\right)=-1 / 3 \log K_{8}+\log f\left(S_{2}\right) \log K_{8}=61.715$ pour $500^{\circ} \mathrm{C}$. 
(9) $\mathrm{Mn}_{8} \mathrm{Be}_{6} \mathrm{Si}_{6} \mathrm{O}_{24} \mathrm{~S}_{2}+\mathrm{SiO}_{2}+\mathrm{O}_{2} \rightarrow 4 \mathrm{Mn}_{2} \mathrm{SiO}_{4}+3 \mathrm{Be}_{2} \mathrm{SiO}_{4}+\mathrm{S}_{2}$ $\log f_{\left(\mathrm{O}_{2}\right)}=-\log \mathrm{K}_{9}-\log \left(\mathrm{S}_{2}\right) \quad \log \mathrm{K}_{9}=5.97$ pour $500^{\circ} \mathrm{C}$.

Le domaine de la stabilité de la danalite est défini par les équations (Burt 1980) :

(10) $8 \mathrm{Fe}+3 \mathrm{SiO}_{2}+3 \mathrm{Be}_{2} \mathrm{SiO}_{4}+3 \mathrm{O}_{2}+\mathrm{S}_{2} \rightarrow \mathrm{Fe}_{8} \mathrm{Be}_{6} \mathrm{Si}_{6} \mathrm{O}_{24} \mathrm{~S}_{2}$ $\log f\left(O_{2}\right)=-1 / 3 \log K_{10}-1 / 3 \log f\left(S_{2}\right) . \log K_{10}=108.53$.

(11) $8 \mathrm{FeS}+3 \mathrm{Be}_{2} \mathrm{SiO}_{4}+3 \mathrm{SiO}_{2}+3 \mathrm{O}_{2} \rightarrow \mathrm{Fe}_{8} \mathrm{Be}_{6} \mathrm{Si}_{6} \mathrm{O}_{24} \mathrm{~S}_{2}+3 \mathrm{~S}_{2}$ $\left.\left.\log \mathrm{fO}_{2}\right)=-1 / 3 \log \mathrm{K}_{11}-\log \mathrm{f}_{2}\right) . \quad \log \mathrm{K}_{11}=51.62$.

(12) $8 \mathrm{FeS}_{2}+3 \mathrm{Be}_{2} \mathrm{SiO}_{4}+3 \mathrm{SiO}_{2}+3 \mathrm{O}_{2} \rightarrow \mathrm{Fe}_{8} \mathrm{Be}_{6} \mathrm{Si}_{6} \mathrm{O}_{24} \mathrm{~S}_{2}+7 \mathrm{~S}_{2}$ $\log f\left(O_{2}\right)=-1 / 3 \log K_{12}+7 / 3 \log f\left(S_{2}\right) . \log K_{12}=27.14$

(13) $3 \mathrm{Fe}_{8} \mathrm{Be}_{6} \mathrm{Si}_{6} \mathrm{O}_{24} \mathrm{~S}_{2}+7 \mathrm{O}_{2} \rightarrow 8 \mathrm{Fe}_{3} \mathrm{O}_{4}+9 \mathrm{Be}_{2} \mathrm{SiO}_{4}+9 \mathrm{SiO}_{2}+3 \mathrm{~S}_{2}$ $\log f\left(O_{2}\right)=-1 / 7 \log K_{13}+3 / 7 \log f\left(S_{2}\right) \cdot \log K_{13}=403$

(14) $\mathrm{Fe}_{8} \mathrm{Be}_{6} \mathrm{Si}_{6} \mathrm{O}_{24} \mathrm{~S}_{2}+\mathrm{O}_{2}+\mathrm{SiO}_{2} \rightarrow 4 \mathrm{Fe}_{2} \mathrm{SiO}_{4}+3 \mathrm{Be}_{2} \mathrm{SiO}_{4}+\mathrm{S}_{2}$ $\log f\left(O_{2}\right)=-\log K_{14}+\log \left(S_{2}\right) \quad \log K_{14}=14.07$

Le domaine de stabilité de la genthelvite est délimité par les équations (Burt 1988, Bilal \& Fonteilles 1988, 1991):

(15) $8 \mathrm{ZnS}+3 \mathrm{Be}_{2} \mathrm{SiO}_{4}+3 \mathrm{O}_{2}+3 \mathrm{SiO}_{2} \rightarrow \mathrm{Zn}_{8} \mathrm{Be}_{6} \mathrm{Si}_{6} \mathrm{O}_{24} \mathrm{~S}_{2}+3 \mathrm{~S}_{2}$ $\log f_{\left(O_{2}\right)}=-1 / 3 \log K_{15}+\log f\left(S_{2}\right) \quad \log K_{15}=47.6$

(16) $\mathrm{Zn}_{8} \mathrm{Be}_{6} \mathrm{Si}_{6} \mathrm{O}_{24} \mathrm{~S}_{2}+\mathrm{SiO}_{2}+\mathrm{O}_{2} \rightarrow 3 \mathrm{Be}_{2} \mathrm{SiO}_{4}+4 \mathrm{Zn}_{2} \mathrm{SiO}_{4}+\mathrm{S}_{2}$

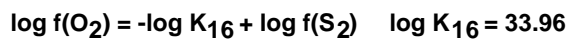

Les domaines de stabilité des autres minéraux associés sont:

(17) $2 \mathrm{Fe}+\mathrm{S} 2 \rightarrow 2 \mathrm{FeS}$

$\log \mathrm{K}=2 \log \mathrm{KFeS}-\left[2 \log \mathrm{K}_{\mathrm{Fe}}+\log \mathrm{K}_{\mathrm{S} 2}\right]$

$\log K=2(9.761)-[2(1.367)+2.56)]$

$\log f\left(S_{2}\right)=-14.22$

\section{(18) $2 \mathrm{FeS}+\mathrm{S}_{2} \rightarrow 2 \mathrm{FeS}_{2}$}

$\log \mathrm{K}=2 \log \mathrm{K}_{\mathrm{FeS} 2}-\left[2 \log \mathrm{K}_{\mathrm{FeS}}+\log \mathrm{K}_{\mathrm{S} 2}\right]$

$\log \left(f_{S 2}\right)=-\log K$

$\log K_{500}=2(14.1)-[2(9.761)+2.56]=6.118$

$\log \left(f_{S}\right)=-6.118$

(19) $2 \mathrm{Fe}+\mathrm{SiO}_{2}+\mathrm{O}_{2} \rightarrow \mathrm{Fe}_{2} \mathrm{SiO}_{4}$

$\log \mathrm{K}=\log \mathrm{K}_{\mathrm{Fe} 2 \mathrm{SiO} 4}-\left[2 \log \mathrm{K}_{\mathrm{Fe}}+\log \mathrm{K}_{\mathrm{SiO} 2}+\log \mathrm{K}_{\mathrm{S} 2}\right]$

$\log \mathrm{K}=76.22-[2(1.367)+60.147+2.56)] \log \mathrm{fO}_{2}=-30.65$

(20) $3 \mathrm{Fe}_{2} \mathrm{SiO}_{4}+\mathrm{O}_{2} \rightarrow 3 \mathrm{SiO}_{2}+2 \mathrm{Fe}_{3} \mathrm{O}_{4}$

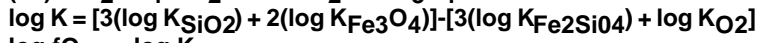
$\log \mathrm{fO}_{2}=-\log \mathrm{K}$

$\log \mathrm{K}_{500}=[2(76.22)+3(60.147)]-[3(100.653)+7.124]=23.797$

$\log \mathrm{fO}_{2}=-23.797$

(21) $4 \mathrm{Fe}_{3} \mathrm{O}_{4}+\mathrm{O}_{2} \rightarrow 6 \mathrm{Fe}_{2} \mathrm{O}_{3}$

$\log \mathrm{K}=6 \log \mathrm{KFe}_{\mathrm{Fe}} \mathrm{O} 3-\left[4 \log \mathrm{KFe}_{\mathrm{Fe}} \mathrm{O}+\log \mathrm{KO} 2\right.$
$\log \mathrm{K}=6(55.132)-[4(76.22)+7.124]$

$\log \mathrm{O}_{2}=-18.79$

Dans les dépôts à sulfure, la phénacite est tardive, elle se développe sur l'helvite. Par contre, dans les formations où les sulfures n'ont qu'un rôle secondaire c'est la fugacité d'oxygène qui contrôle la stabilité de la phénacite.

La danalite cristallise dans un domaine de fugacité de soufre et d'oxygène très limité (Fig. 6) qui correspond au domaine de stabilité de la pyrrhotite. La genthelvite est stable dans un large domaine de fugacité d'oxygène et de soufre. Par contre l'helvite se développe dans un domaine de fugacité d'oxygène modéré et de fugacité de soufre assez large comparable au domaine de la genthelvite.

Etant donné la plus forte affinité du soufre pour Zn que pour Fe, si on élève la fugacité de soufre (Fig. 6) on va tendre à sortir du domaine de la genthelvite pure avant de sortir de celui de la danalite.

Le remplacement de la danalite par la genthelvite pourrait théoriquement se produire suivant la réaction (22):
(22) $\mathrm{Fe}_{8} \mathrm{Be}_{6} \mathrm{Si}_{6} \mathrm{O}_{24} \mathrm{~S}_{2}+8 \mathrm{ZnS}+4 \mathrm{~S}_{2} \rightarrow \mathrm{Zn}_{8} \mathrm{Be}_{6} \mathrm{Si}_{6} \mathrm{O}_{24} \mathrm{~S}_{2}+8 \mathrm{FeS}_{2}$

Si nous continuons à élever la fugacité du soufre, on va détruire la genthelvite suivant la réaction (15) mais à cause de l'affinité de Zn pour le soufre la réaction (11) de destruction de la danalite se produit avant la réaction (15). Donc la genthelvite ne pourra pas remplacer la danalite à fugacité de soufre croissante et la réaction (22) ne se produit pas.

A Sucuri les ilménites de la zone interne (où se développe la genthelvite) ont $16 \%$ d'hématite en solution solide. Dans la zone externe, les ilménites sont dépourvues de constituant hématitique. La zonation des helvites (un coeur de genthelvite et une bordure de danalite) traduit une baisse de fugacité d'oxygène et une augmentation de celle de soufre.

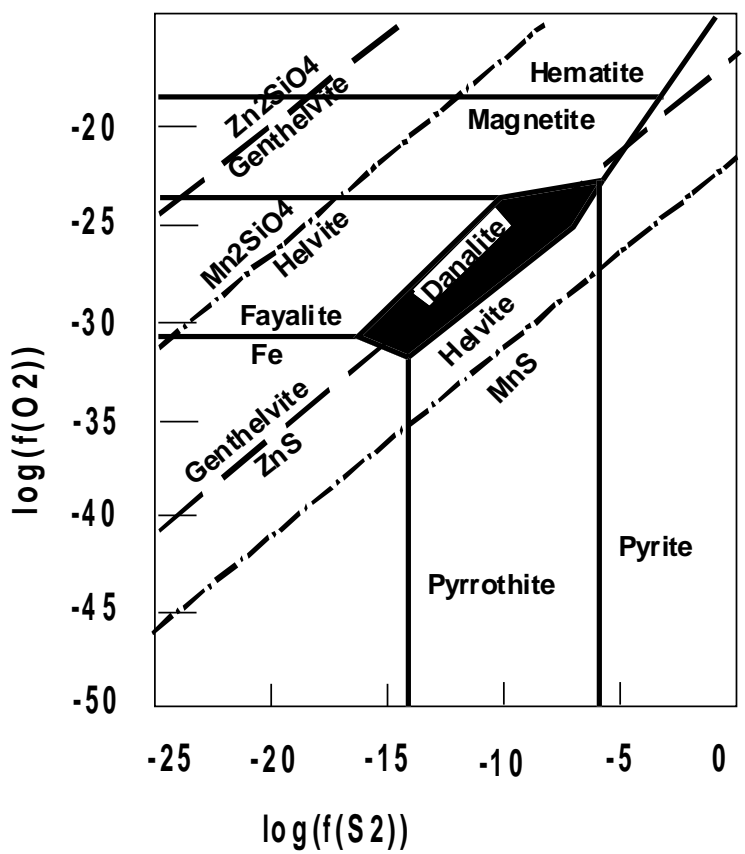

Figure 6 : Les domaines de stabilité des minéraux du groupe de l'helvite dans le diagramme fugacité d'oxygène en fonction de la fugacité de soufre. Les données thermodynamiques utilisées sont de Robie et al. (1979) et de Fursenko (1989). Ce diagramme est légèrement différent de celui de Fursenko (1989) pour la même température.

Figure 6: Stability fields of helvite group minerals in the $f\left(\mathrm{O}_{2}\right)$ versus $f\left(\mathrm{~S}_{2}\right)$ diagram. Thermodynamic data after Robie et al. (1979) and Fursenko (1989).

CONTRAINTES GÉOCHIMIQUES DU MILIEU: Les études des inclusions fluides dans le quartz, béryl et helvite indiquent des fluides aqueux (la phase aqueuse représente plus de $70 \%$ du volume total de l'inclusion) de concentration en sels dissous de $13 \%$ à $26 \%$ en poids équivalent $\mathrm{NaCl}(13 \%$ à $25 \%$ à Costabonne (Guy 1988), $18 \%$ à $25 \%$ à Sucuri (ce travail) et $26 \%$ à Sunnyside (Casadevall \& Ohmoto 1977). L'ion dominant en solution est toujours le $\mathrm{Na}^{+}$.

Le béryllium est facilement transporté par les solutions acides et faiblement alcalines mais sa mobilité diminue rapidement si l'alcalinité de la solution augmente. Cependant, en présence de calcite 
le Be reste en solution si la teneur en $\mathrm{Na}$ ou $\mathrm{K}$ est élevée. Newkirk (1969) a en effet montré que la solubilité du Be croît avec la concentration en Na. Par contre en présence de $\mathrm{Mg}$ (dolomite) le Be se dépose (Soboleva et al. 1972).

Dans les greisens, les albitites, les veines à quartz et les skarns on rencontre l'association de minéraux béryllifères et fluorifères

Le béryl est le minéral du béryllium le plus commun dans les greisens et dans les veines à sidérophyllite ou à quartz. Il est stable dans des conditions relativement acides correspondant au tampon muscovite, quartz et feldspath. Dans la veine à sidérophyllite (Sucuri) la cristallisation du grenat (liée à une forte activité de $\mathrm{Mn}$ ) a favorisé la formation de la phénacite au lieu du béryl suivant la réaction:

$2 \mathrm{Be}_{3} \mathrm{Al}_{2} \mathrm{Si}_{3} \mathrm{O}_{18}+6 \mathrm{MnO}+3 \mathrm{SiO}_{2} \rightarrow$

$2 \mathrm{Mn}_{3} \mathrm{Al}_{2} \mathrm{Si}_{3} \mathrm{O}_{12}+3 \mathrm{Be}_{2} \mathrm{SiO}_{4}+6 \mathrm{O}_{2}$

Une forte activité de la silice ou du manganèse provoquerait le déplacement de l'équilibre vers la droite (phénacite et grenat).

Fursenko (1989) a montré expérimentalement à $600^{\circ} \mathrm{C}$ et $1 \mathrm{~kb}$ qu'au fur et à mesure de la diminution de la valeur du rapport molaire $\mathrm{Mn} / \mathrm{Al}$ de la solution (Fig. 7), et en présence de soufre, nous avons la suite paragénétique suivante : helvite et phénacite, $\mathrm{MnS}$ et phénacite, béryl et phénacite et finalement béryl seul.

A Dajishan dans la veine à quartz et muscovite, la muscovite primaire est détruite au profit des feldspaths et de la biotite. Ce stade est marqué par une forte activité du calcium (grenat plus calcique) et une forte activité du manganèse (à cause de la transformation de la wolframite en scheelite). Le béryl, qui appartient à la phase primaire (muscovite, quartz, grenat et felsdpaths), n'est plus stable. L'helvite associée aux grenats calciques et à la scheelite cristallise suivant la réaction (7).

Dans les skarns de Costabonne par exemple c'est l'évolution du rapport $\mathrm{Si} / \mathrm{Al}$ durant le développement de la skarnification qui est, à notre avis, responsable de la diversité de composition de minéraux de béryllium. Dans les skarns à brucite l'association chondrodite, musgravite $\left(\mathrm{Mg}_{2} \mathrm{BeAl}_{6} \mathrm{O}_{12}\right)$, bromellite $\left(\mathrm{Be}(\mathrm{OH})_{2}\right)$ et spinelle dans les veines à silicate et spinelle de la dolomie témoigne de la faible activité de la silice par rapport à celle de l'alumine. Les deux minéraux du béryllium dans cette zone sont caractéristiques des milieux sous-saturés en silice. Dans les skarns Nord, l'helvite est associée à la phase tardive (amphibole, calcite et quartz) dans la zone de pyroxénite où l'activité de l'alumine est très réduite. La formation des skarns à brucite est antérieure à celle des skarns Nord et elle représente un stade précoce de la skarnification (Guy 1988).

La formation de minéraux de béryllium de composition variable dans les skarns résulterait:

- Soit de la succession de solution tardive riche en silice (le rapport $\mathrm{Si} / \mathrm{Al}$ est fort, la phénacite ou l'helvite cristallise) sur la paragenèse alumineuse (musgravite, chrysobéryl) formée précocement

- Soit de la faible migration de $\mathrm{Al}$ par rapport à $\mathrm{Si}$, et dans ce cas la paragenèse alumineuse (musgravite, chrysobéryl) aura une extension limitée.

Dans les albitites de Sucuri et de Millas (Puyau, communication personnelle) et dans les syénites de Jos et de Lupiko au Nigéria (Burbank 1933), les minéraux du béryllium les plus communs sont la phénacite et ceux du groupe de l'helvite.

Dans les veines à quartz (Sucuri et Dajishan), la présence du béryl et de l'helvite, et/ou de la phénacite, est le résultat de l'action d'une solution tardive alcaline (feldspathisante) sur la paragenèse primaire plus alumineuse à quartz, muscovite et béryl.

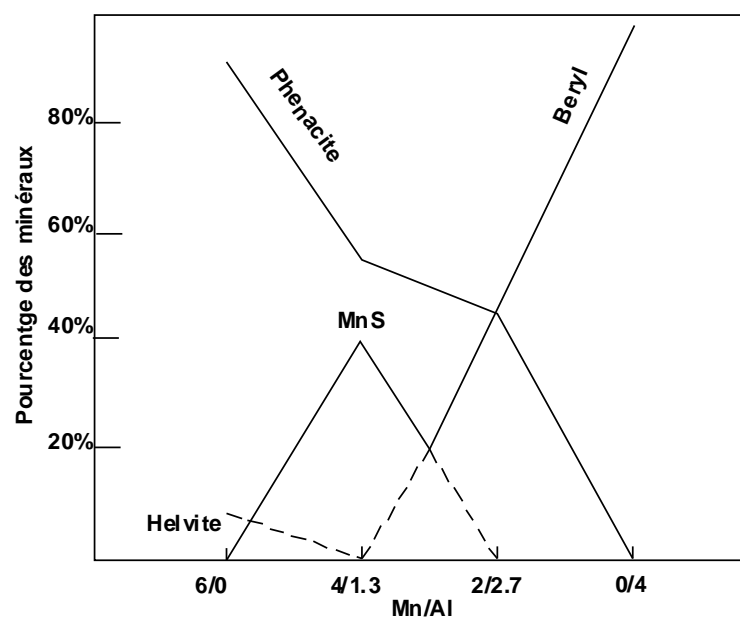

Figure 7: Relation entre les minéraux du groupe de l'helvite et le rapport Mn/Al dans les fluides Fursenko (1989).

Figure 7: Relation between helvite group minerals and $M n / A l$ ratio in the fluids.

L'activité de l'alumine joue un rôle essentiel sur la variation de la composition des minéraux du béryllium. Si elle est faible la phénacite et/ou l'helvite sont stables et si elle est forte, le béryl, le chrysobéryl ou la musgravite se trouvent stabilisés. Cependant l'augmentation de l'activité de certains éléments $(\mathrm{Ca}$, $\mathrm{Mn}, \mathrm{Fe}, \mathrm{Zn}$, ou des alcalins) tendant à incorporer l'alumine dans les grenats ou dans les feldspaths sera défavorable à la formation du béryl. Ainsi l'helvite est typiquement un minéral stable dans les skarns calciques ou magnésiens et il s'associe à la phénacite dans les roches affectées par un métasomatisme alcalin. En revanche, le béryl apparaît normalement dans les conditions où le fluide de caractère acide se sépare des liquides granitiques très évolués (aplopegmatites).

La genthelvite est favorisée par rapport aux autres variétés du groupe de l'helvite par l'alcalinité du fluide (albitite de Sucuri, syénite de Lupiko (Burbank 1933). En effet, les travaux expérimentaux de Fursenko (1989) à $600^{\circ} \mathrm{C}$ et $1 \mathrm{~Kb}$ ont montré que la genthelvite est le minéral le plus stable du groupe de l'helvite dans les solutions alcalines où le rapport molaire 
$\mathrm{NaOH} / \mathrm{HCl}$ est supérieur à 1 (Fig.8). Lorsque ce rapport augmente dans le fluide la proportion du pôle genthelvite croît.

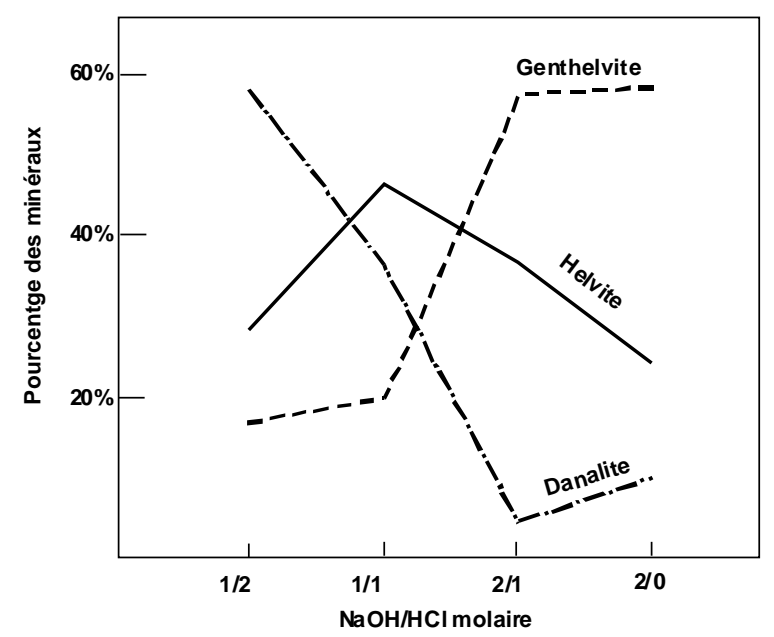

Figure 8: Relation entre les minéraux du groupe de l'helvite et le rapport $\mathrm{NaOH} / \mathrm{HCl}$ du fluide d'après Fursenko (1989).

Figure 8: Relation between helvite group minerals and $\mathrm{NaOH} / \mathrm{HCl}$ ration in the fluid.

La zonation de l'helvite dans les albitites de Sucuri (un coeur de genthelvite et une bordure de danalite) traduit donc une diminution de l'alcalinité au fur et à mesure de l'évolution du fluide. Cette zonation à l'échelle du cristal est également présente à l'échelle de l'affleurement. La composante genthelvite dans la danalite de l'albitite du massif de Sucuri diminue depuis la zone interne $\mathrm{Ge}_{64} \mathrm{Da}_{10} \mathrm{He}_{26}$ jusqu'à la zone externe $\mathrm{Ge}_{23} \mathrm{Da}_{50} \mathrm{He}_{26}$. Nous observons la même variation de la composante genthelvite dans la danalite en bordure de la veine à sidérophyllite albitisée $\mathrm{Ge}_{25} \mathrm{Da}_{42} \mathrm{He}_{33}$ (zone faiblement albitisée) et $\mathrm{Ge}_{55} \mathrm{Da}_{39} \mathrm{He}_{22}$ (dans la zone fortement albitisée).

Nous pouvons tenter d'écrire la composition du pôle de la genthelvite sous la forme $\mathrm{Zn}_{8-\mathrm{x}} \mathrm{Na}_{\mathrm{x}} \mathrm{Be}_{6} \mathrm{Si}_{6} \mathrm{O}_{24} \mathrm{~S}_{2-\mathrm{x}} \mathrm{Cl}_{\mathrm{x}}$, nous avons l'équilibre (23) :

(23) $7.81 \mathrm{ZnS}+3 \mathrm{Be}_{2} \mathrm{SiO}_{4}+0.19 \mathrm{NaCl}+3 \mathrm{SiO}_{2}+4 \mathrm{O}_{2} \rightarrow$

$$
\mathrm{Zn}_{7.81} \mathrm{Na}_{0.19} \mathrm{Be}_{6} \mathrm{Si}_{6} \mathrm{O}_{24} \mathrm{~S}_{1.81} \mathrm{Cl}_{0.19}+3 \mathrm{~S}_{2}
$$

Le déplacement de cet équilibre dépend du degré de l'alcalinité et de la fugacité de soufre.

Les variations de composition des minéraux de l'helvite dans le massif de Sucuri (Fig. 2) résulteraient des variations de la fugacité de soufre et de celle de l'oxygène à l'intérieur du fluide. La genthelvite est stable dans des conditions alcalines et oxydantes.

\section{CONCLUSION}

L'activité de l'alumine joue un rôle essentiel sur la variation de la composition des minéraux du béryllium. Si elle est faible la phénacite et/ou l'helvite sont stables et si elle est forte le béryl, le chrysobéryl ou la musgravite se trouvent stabilisés. Cependant l'augmentation de l'activité de certains éléments $(\mathrm{Ca}$,
$\mathrm{Mn}, \mathrm{Fe}, \mathrm{Zn}$, ou des alcalins) tendant à incorporer l'alumine dans les grenats ou dans les feldspaths sera défavorable à la formation du béryl.

L'étude paragénétique, géochimique et expérimentale des helvites rencontrées dans ces gisements aboutit à une nouvelle définition de la formule structurale de l'helvite par analogie à celle de la haüyne. Nous pouvons proposer la formules suivante: (Mn, $\mathrm{Zn}, \mathrm{Fe})_{8-\mathrm{x}} \mathrm{Na}_{\mathrm{x}} \mathrm{Be}_{6} \mathrm{Si}_{6} \mathrm{O}_{24} \mathrm{~S}_{2-\mathrm{x}} \mathrm{Cl}_{\mathrm{x}}$.

La danalite (pôle ferrifère de l'helvite) cristallise dans un domaine de fugacité de soufre et d'oxygène très limité qui correspond au domaine de stabilité de la pyrrhotite. La genthelvite (pôle zincifère de l'helvite) est stable dans un large domaine de fugacité d'oxygène et de soufre. Par contre l'helvite (pôle manganifère de l'helvite) se développe dans un domaine de fugacité d'oxygène modéré et de fugacité de soufre assez large comparable au domaine de la genthelvite. La zonation des helvites (un coeur de genthelvite et une bordure de danalite) traduit une baisse de fugacité d'oxygène et une augmentation de celle de soufre.

La composante genthelvite dans la danalite des albitites diminue de la zone interne $\mathrm{Ge}_{64} \mathrm{Da}_{10} \mathrm{He}_{26}$ jusqu'à la zone externe $\mathrm{Ge}_{23} \mathrm{Da}_{50} \mathrm{He}_{26}$. Nous observons la même variation de la composante genthelvite dans la danalite en bordure de la veine à sidérophyllite albitisée $\mathrm{Ge}_{25} \mathrm{Da}_{42} \mathrm{He}_{33}$ (zone faiblement albitisée) et $\mathrm{Ge}_{55} \mathrm{Da}_{39} \mathrm{He}_{22}$ (dans la zone fortement albitisée). La genthelvite est favorisée par rapport aux autres variétés du groupe de l'helvite par l'alcalinité du fluide.

\section{REMERCIEMENTS}

Ce travail a été réalisé dans le cadre de coopération entre le CNRS/CNPq, l'Université de Brasilia et l'Ecole des Mines de Saint-Etienne. Nous remercions ces organismes pour leur aide.

\section{REFERENCES BIBLIOGRAPHIQUES}

AHRENS, L.H. 1952. The use of ionization potentials. Part I. Ionic radii of the elements. Geochim. Cosmochim. Acta- 2, 155-169. ANDRADE, G.F. \& DANNI, J.C.M. 1978. As mineralizações de estanho, berílio e cobre do Granito Serra Branca, Cavalcante, GO. In: CONG. BRAS. GEOL., 30. Recife, 1978. Anais..., vol.6, Recife, SBG. p. 2571-2593.

BARTH, T.F.W. 1926. Die kristallographische beziehung zwischen helvin and sodalit. Norsk Geologisk Tidsskrift- 9, 40-42.

BEUS, A.A. 1962. Beryllium. Freeman and $C^{\circ}$, San Francisco. - 160

BILAL, E. 1991. Etude de deux massifs granitiques de la province stannifère de l'état de Goias (Brazil) et des formations métasomatiques associées aux minéralisations en Sn et Be. Paris, 480p. Thèse de Doctorat, Ecole de Mines de Paris.

BILAL, E. \& FONTEILLES, M. 1988. Conditions d'apparition respectives de l'helvite, de la phénacite et du béryl dans l'environnement granitique : exemple du massif de Sucuri (Brésil). C.R. Acad. Sci. Paris, t. 307, série II: 273-276.

BILAL, E. \& FONTEILLES, M. 1991. Chemical properties of helvite group minerals in different types of occurences. In Source, Transport and Deposition of Metals (M. Pagel \& J.L. Leroy, ed.). Balkema, Rotterdam, The Netherlands. 733-736.

BURBANK, W.S. 1933. Manganese minerals of the Sunnyside veins, Eureka Gulch, Colorado. Am. Mineral., 18 (12): 513-527.

BURT, D.M. 1980. The stability of danalite $\mathrm{Fe}_{3} \mathrm{Be}_{3}\left(\mathrm{SiO}_{4}\right)_{3} \mathrm{~S} . A m$. Mineral., 65: 355-360. 
BURT, D.M. 1988. Stability of genthelvite, $\mathrm{Zn}_{3} \mathrm{Be}_{3}\left(\mathrm{SiO}_{4}\right)_{3} \mathrm{~S}$ : an exercise in chalcophilicity using exchange operators. Am. Mineral., 73: 1384-1394.

CASADEVALL, T. \& OHMOTO, H. 1977. Hunnyside Mine, Eureka Mining District, San Juan County, Colorado: geochemistry of gold and base Metal Ore deposition in a volcanoe environment. Econ. Geol.- vol. 72,1285-1320.

COLLINS, P.L.F. 1979. Gas hydrates in $\mathrm{CO}_{2}$ - bearing fluid inclusions and the use offreezing data for estimation of salinity. Econ. Geol.74, 1435-1444.

DUBRU, M. 1986. Pétrologie et géochimie des marbres à brucite et des borates associés au gisement de tungstène de Costabonne (Pyrénées Orientales, France). Thèse Doctorat Université Catholique de Louvain.- 443 p.

DUMONCEAU, J.; BIGOT, S.; TREUIL, M.; FAUCHERRE, M. \& FROMAGE, F. 1979. Détermination des constantes de formation des tétracarbonato-lanthanides (III). Rev. Chimie Minérale-16, 583-592.

DUNN, P.J. 1976. Genthelvite and the helvite group. Mineral. Mag., 40: $627-636$

FLYNN, R.T. \& BURNHAM, C.W. 1978. An experimental determination of rare earth partition coefficients between a chloride containing vapor phase and silicate melts. Geochim. Cosmochim. Acta- 42, 6A, 685-701.

FONTEILLES, M.; GUY, B. \& SOLER, P. 1978. The influence of wall-rock on skarn mineralization at the Sala and Costabonne tungsten deposits (Pyrenees, France). Geol. Soc. Am. Abstr. Programs- 10, 3, 105.

FONTEILLES, M.; GUY, B. \& SOLER, P. 1980. Etude du processus de formation des gites de skarns de Salau et Costabonne. Mémoire BRGM.- n 99 , ch. 7, 259-282.

FONTEILLES, M.; RAIMBAULT, L. \& FOUILLAC, A.M. 1987. Magmatic history and geochemical characteristics of granites associated with Dajishan tungsten deposit. International Symposium on Petrogenesis and Mineralization of Granitoids, Guangzhou.- 78-79.

FONTEILLES, M.; RAIMBAULT, L. \& FOUILLAC, A.M. 1990. The granites associated with Dajishan tungsten deposit. Proceedings of the International Symposium on Petrogenesis and Mineralization of Granitoids.

FURSENKO, D.A. 1989. Uslovija sinteza mineralov gruppy gel'vina. Izd. Nauka, Sibirskoe Otdelenie, Novosibirsk, 76 p.

GLASS, J.J.; JAHNS, R.H. \& STEVENS, R.E. 1944. Helvite and danalite from New Mexico and the helvite group. Am. Mineral.29, 163-191.

GOLDSCHMIDST, V.M. 1911. Die Gesetze der Mineralassociation von standpunkt der Phasenregel. Z. Anorgan. Chem., 71: 313322 .

GUITARD, G. \& LAFFITE, P. 1958. Les calcaires métamorphiques et les skarns du Pic de Costabonne (Pyrénées Orientales). Sc. Terre Nancy. - IV, 1-2, 57-137.
GUY, B. 1988. Contribution à l'étude des skarns de Costabonne (Pyrénées Orientales, France) et à la théorie de la zonation métasomatique. Thèse de Doct. ès Sciences, Université P.\& M. Curie, Paris, $480 \mathrm{p}$.

HASSAN, I. \& GRUNDY, H.D. 1984. The crystal structures of sodalite-group of minerals. Acta Crystallographica B40: 6-13.

HASSAN, I. \& GRUNDY, H.D. 1985. The crystal structures of helvite group minerals, $(\mathrm{Mn}, \mathrm{Fe}, \mathrm{Zn})_{8} \mathrm{Be}_{6} \mathrm{Si}_{6} \mathrm{O}_{2} 4 \mathrm{~S}_{2}$. Amer. Mineral., 70: 186-192.

IRVING, A.J. \& FREY, F.A. 1978. A review of experimental studies of crystal/liquid trace element partitioning. Geochim. Cosmochim. Acta. - 42, 743-770.

MINEYEV, D.A. 1963. Geochemical differenciation of the rare earths. Geochemistry (URSS)- 12, 1129-1149.

MOOKHERJEE, A. 1962. Certain aspects of the geochemistry of cadmium. Geochim. Cosmochim. Acta- 26, 351-360.

NEWKIRK, H.W. 1969. The system beryllium oxide-water at moderate temperatures and pressure. Inorg. Chem.- 3, 10411043.

PIMENTEL, M.M.; HEAMAN, L.; FUCK, R.A. \& MARINI, O.J. 1990. U-Pb zircon geochronology of precambrian tin-bearing continental type acid magmatism in eastern Goias, Brazil. Precambrian Research, 52: 321-335.

RAGU, A. 1994. Helvite from the french pyrenees as evidence for granite related hydrothermal activity. The canadian mineralogist,32, 111-120.

RAIMBAULT, L.; FONTEILLES, M. \& FOUILLAC, A.M. 1987. REE pattern evolution in the fluid phase and vein mineral composition in Dajishan tungsten deposit. International Symposium on Petrogenesis and Mineralization of Granitoids, Guangzhou, 250-251.

ROBIE, R.A.; HEMINGWAY, B.S. \& FISHER, J.R. 1979. Thermodynamic Properties of Minerals and Related Substances at $298.15 \mathrm{~K}$ and $1 \mathrm{Bar}$ (105 Pascals) Pressure and at Higher Temperatures. Geological Survey Bull., nº 1452, 456 p.

SCOTT, S.D. \& BARNES, H.L. 1971. Sphalerite geothermometry and geobarometry. Econ. Geol.- 66, 653-669.

SOBOLEVA, G.I.; TURGARINOV, I.A. \& KHITAROV, D.N. 1972. The role of calcium and magnesium in chrysoberyl mineralization. Geo. Int., 9, 944-947.

VLASOV, K.A. 1966. Geochemistry of rare elements. Israel Program for Scientific Translations, 688 p. Translated from Geokhimija Redkikh Elementov, Izd. Nauka, Moskva, 1964.

WALENTA, V.K. 1981. Grüner Helvin von Azegour in Marokko. Aufschluss, 32, 443-449.

WIGGINS, L.B. \& CRAIG, J.R. 1980: Reconnaissance of the CuFe-Zn-S system : Sphalerite phase relationships. Econ. Geol., 75: $742-751$. 
